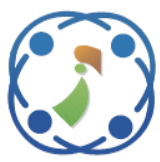

\title{
Throughput Enhancement of the Edge User Equipments Based on the Power- Bandwidth Tradeoff in the Optical Attocell Networks
}

\author{
Abdelmoujoud Assabir ${ }^{1 *}$ \\ Jamal Elmhamdi ${ }^{1}$ \\ Ahmed Hammouch ${ }^{2}$ \\ ${ }^{1}$ Mohammed V University-ENSET, Rabat, Morocco \\ ${ }^{2}$ Administration of Scientific Research and Innovation at the Ministry of National Education and Vocational \\ Training and Higher Education and Scientific Research Rabat, Morocco \\ * Corresponding author's Email: abdelmoujoud.assabir@um5s.net.ma
}

\begin{abstract}
Indoor communication system has known an exponentially growth due to high demand of wireless data service ratio. As an alternative way, the visible light zone of the electromagnetic spectrum is explored to fulfil the future wireless data traffic requirements. Among the main issues that can be handled in the visible light communication (VLC) system are the resources allocation and interferences management. In this paper, we aim to enhance the throughput of the edge user based on the joint optimization of the power and bandwidth in the VLC downlink indoor cellular network. We propose a new geometry design that alleviates the inter-cell interference (ICI) of the edge users which occurs especially in the overlapping areas between the adjacent cells. An orthogonal frequency division multiple access (OFDMA) based on the direct current optical (DCO) is regarded as the channel access protocol for supporting the multiusers at the same time. At the edge of the coverage area, we evaluate the performance of the proposed method in terms of the achievable throughput and signal-to-interference-plus-noise ratio (SINR).Furthermore, based on the least square (LS), the joint optimization of the power and bandwidth result to a parameter $\rho_{0}$ which varies between 0 and 1 . The results show that the new optimization with $\rho_{0}=0.9$ depict better improvements in term of throughput $\mathrm{R}\left(\rho_{0}\right)_{\mathrm{D}, \delta}=65 \mathrm{Mbps}$ at $\mathrm{r}_{3,1}=3 \mathrm{~m}$. We further notice that the signal-noise-ratio (SNR) is increased with $\rho_{0}=0.9$ at the expense of the error probability falling $\left(\mathrm{P}_{\mathrm{z}} \cong 10^{-4}\right)$.
\end{abstract}

Keywords: Visible light communication, Inter-cell interference, Orthogonal frequency division multiple access, Direct current optical, Signal-to-interference-plus-noise ratio, Least square, Signal-noise-ratio.

\section{Introduction}

The increasing demand crunch for wireless capacity results in a high limit in the radio frequency spectrum. Compared to 2016, the numerical statistics which has been shown recently by Cisco (Feb 7, 2017), they will be sevenfold per month by 2021 in the global mobile data traffic [1]. Along with the evolution of the social applications such as Facebook, Twitter..., 74 percent of the growth of the latter (data traffic) will be expected to be found over the next five years. As well known, the radio frequency spectrum suffers from several issues like interference as the main problem, bandwidth limitation, security issues, and so on. Therefore, the next future alternative should be able to offer higher capacity and overcome the various drawbacks coming from the conventional communication system. Light-fidelity (LI-FI) and VLC systems have been considered as a promising alternative technology and a subset of the optical wireless channel (OWC). They can also resolve performance limitation due to bandwidth insufficiency and achieve a high data rate compared to radio frequency (RF) range [2]. Both systems employ the alternative or other region of the electro spectrum reserved for visible light from $380 \mathrm{~nm}$ to $750 \mathrm{~nm}$ (wavelength) corresponding to a frequency spectrum of $430 \mathrm{THz}$ to $790 \mathrm{THz}$, whereas in VLC system a unidirectional communication is considered to be in downlink based on visible light for data transmission. However, in LI-FI system a fully networked bidirectional technique is proposed based on the 
visible light in downlink and infrared (IR) in the uplink [3]. OWC has the immunity to security issues that appear in the RF communication system. Moreover in electromagnetic spectrum based on RF range, the signal is diffused in all directions that produce the interference within the receiving range, while in VLC system, the optical signal coming from the light emitting diode (LED) is significantly based on intensity modulation (IM) with direct detection (DD). OWC has also known noticeable advances from academia and industry due to the growing number of portable devices and user terminals indoor, by applying the idea of using the LED as transmitter for both illumination and communication where positive-intrinsic-negative photodiode (PIN-PD) or avalanche photodiode (APD) as receivers. The LED is anticipated to be the fundamental source in the solid state-lighting, and has opened the door for applying a new communication system as mentioned above. Compared to other sources of artificial light like the incandescent light bulbs, the LED offers several advantages such as low power consumption, low cost, long lifetime, high brightness, no health hazards, data security and high level of power efficiency. Therefore, the LED suffers from two main issues: nonlinearity due to short dynamic range of its behavior and limitation of the maximum achievable data rate owing to available modulation bandwidth. Thus, lots of researches have been carried out to handle both problems by relying on postdistortion to linearize the nonlinear portion of the LED and expand the bandwidth of the LED by filtering out the phosphor element of the optical power [4]. Many prospective applications such as LI-FI, communication vehicle-to-vehicle (V2V) vehicle-to-infrastructure (V2I) and submarine communication have been investigated by researchers [5]. In Fig. 1 (a), where one of the most challenges is shown to describe the LI-FI proposed technology that will be integrated in the future railway system. Moreover, two main units will probably drive optical $\mathrm{LiFi}$ attocell network. The first unit is the substation (SS) that is responsible for both supplying and controlling the continuity of the current in the train lines. This latter will be equipped by the high network devices and modulators in order to carry the data through power-line-communication (PLC) technique simultaneously for using alternative current (AC) in electric power transmission. An asynschrone engine is used to represent the train, the light fixture below the passengers will be harnessed as access point (AP) to serve multiple users equipments (UE). The second unit includes bridge rectifier to convert AC/DC, filtering to extract the data and amplification. The VLC can be involved in the context of automotive communication network for improving road safety, path change alert to avoid accidents. In addition, the IEEE 802.15 has created two standardizations: wireless access in vehicular environments (WAVE) and task group for short range (TG 7r1). The former allows to exchange and use the information between the existing presence of vehicle lights and traffic signal. The latter includes LI-FI, IF and near ultraviolet.

Among the main stringent performance requirements in the wireless communication system is to solve the signal distortion produced by the frequency selective fading of the wireless channel and provides a high spectral efficiency. In that case, orthogonal frequency division multiple (OFDM) is regarded to be a promising candidature in the VLC system and for the next generation wireless communication. By optimizing system performance based on a suitable resources allocation designing, multiple access or OFDMA scheme can be readily used to serve multiuser diversity in an idealistic indoor like offices, airports, and hospitals where different users can employ diverse subcarriers within VLC cells. Meanwhile, AP can be considered as light of the resource allocation (RA) to assign the appropriate subcarriers, power and rate evenly among different UEs. Moreover, the management of the RF resource is built upon the hierarchical structure which will be used in the context of the LI-FI system. In addition, the problem of the rational use of RA, distributions of the users and their position in the illumination zones and the allocation of both power and bandwidth among the existing users becomes an extremely crucial issue that should be well addressed in order to ensure communication quality and improve the system performance. Some of these aforementioned problems have been handled by paving the way to the RA approaches [6], where in [7], S. k. Wilson and J. Holliday adopted a scheduling technique for downlink asymmetrically-clipped optical OFDMA (ACO-OFDMA) which considered the low-pass filter effect of the channel. The latter was relied on two steps: the clusters selection in an OFDMA based on a variable clusters size and the randomizing of a user's cluster selection from a set of clusters. By reducing the information feed-back, the bidirectional user throughput has been enhanced through using two techniques: limited-content feedback and limited-frequency feedback, whereas D. S. Mohammad, X. Wu, M. Safari and H. Haas, in [8] have taken into account the requested data rate and the link quality of the UE for allocating the number of the subcarriers. P. Pattanayak and P. Kumar have discussed in [9] two proposed SINR-based limited feedbacks scheduling algorithms by evaluating their throughput and feedback load performances, where their quality information lies in the form of SINR. 
Furthermore, D. Bykhovsk and S. Arnonin in [10] designed a resource allocation algorithm for managing the interference constrained subcarrier which depends on the allocation of subcarriers to receivers and power allocation for subcarriers in a heuristic way. In order to maximize the system throughput under SINR, a novel joint scheduling and rate allocation algorithm have been developed in [11] by R. K. Mondal, N. Saha, N. T. Le and Y. M. Jang to solve adjacent cells problems which influence transmission link. Additionally, F. Jin, X. Li, R. Zhang, C. Dong and L. Hanzoin in [12], remedied the effect of the interference in the VLC environment applying two interference avoidance approaches: frequency reuse (FR) and vectored transmission (VT), which reduced the interference and hence led to a better performance than the conventional frequency reuse. Finally, the quality of service (QoS) was also regarded in VLC system as a tool to verify to what extent the bandwidth is reduced, whereas M. Z. Chowdhury, M. Shahin Uddin, and Y. Mi. Jang, in [13] proposed a QoS based on a dynamic channel allocation scheme by giving the priority to the higher traffic classes along with reducing the calls blocking probability.

In a cellular typical indoor system, many adjacent APs are deployed in a ceiling to cover the illumination in the entire indoor area, thereby resulting in the overlap between them. ICI is a strong factor which can considerably degrade the performance of the VLC cellular system. As shown in Fig. 1 (b) a typical VLC cellular diffusion is presented with multiuser (black point) scenario which is located in different spaces and two APs are placed in the center of each circle which cover the illumination area and are usually referred to as a cell. The green space represents the inter-cell part. It suffices to state that a lot of studies were predominantly behind the mitigation of the ICI for VLC network [14, 15]. In an aircraft cabin, B. Ghimire and $\mathrm{H}$. Haas compared the performance of the suggested busy burst (BB) approach against chunk allocation by relying on a self-organizing interference management $[14,15]$. The fractional frequency reuse (FFR) was adopted in a DCO attocell network where H. Kazemi and H. Haas improved the average spectral efficiency and downlink SINR performance in the cell edge based on both strict and soft FFR schemes [16]. Afterwards, H. Kazemi, M. Safari and H. Haas proposed in [17] a new spectral efficient cooperative transmission for downlink indoor Li-Fi cellular networks scenario relying on two cooperation protocols: non-orthogonal decode and-forward (NDF) and joint-transmission with decode and-forward (JDF). In [18], L. Chen, W.
Wang and C. Zhang analyzed the interference management at the expense of proposing a coalition formation framework in the VLC context in which the APs are set to self-organize into cooperation coalitions for interference cancellation by using orthogonal time or frequency domain .Finally, A.A.Dowhuszko and A.I. Pérez-Neira in [19] proposed three different transmission schemes in terms of allocating the communication resources , while the data rate was compared with each transmission method.

As aforementioned, co-channel interference (CCI) is one of the main issues that impacts the user performance in an optical attocell network which is caused by using the same subcarrier in neighboring APs at the same time. Therefore, several solutions have been analyzed as mentioned above to mitigate CCI in cellular network including static resource partitioning for mitigating CCI in a cellular optical wireless, optical femtocell system that uses different wavelengths in neighboring cells, and traditional FFR technique that is the cost efficient process to solve the problem of the CCI effectively and to improve both average spectral and cell edge.

In this paper, we exploit both the weakest received signal at the edge of the cell and the interference management which arise when the optical attocell networks is applied by relying on the resource allocation and interference mitigation problems. To handle this technical issue in a smooth manner different than what was mentioned above, a joint optimization (power-band) is used to maximize the throughput of the users at the cell edge based on LS approach. A detailed description of the suggested FFR design will be subsequently analyzed. However; a close look at the main contribution can be summarized as follows: A multiple access is introduced to be a system level downlink VLC scenario based on DCO-OFDMA. Besides, this study takes into account several main problems related to the calculation of the SINR like channel characteristics and cell radius. The coverage area is considered to be hexagonal (HEX) deployment as an idealistic cellular shape compared to the poisson point process (PPP) that represents lower structure of the cellular deployment. We next propose the steps of sharing the OFDMA bandwidth resources in the optical cellular system relying on the resource allocation procedure. In doing so, we divide the lighting center cell area into three non -overlapping regions based on FFR method. We also treat the influence of the various system parameters, encompassing the existence of interfering cells and illumination constraints. 
Before we get into the rest of the paper, we notice that the usefulness behind using the OFDMA manifested in the entire bandwidth is separated into narrow carries, whereas each carrier is allocated to a user and reused in another cell. Additionally, OFDMA eliminates the channel selectivity in both time and frequency over the individual subcarriers and it helps a communication transmission in time which enhances the illumination requirements. However, another multiple access can be used like time division multiple access (TDMA) and code division multiple access (CDMA) by thoroughly considering the allocated time slots and the code, instead of the narrow bands as it will be depicted later. On the one hand, J. Fakidis, D. Tsonev and $\mathrm{H}$. Haas presented in [20] that the OFDMA shows better results along with the user needs data communication and illumination simultaneously in the typical indoor downlink framework. On the other hand, concerning the CDMA, interference averaging is a crucial issue, where the spreading codes are not completely orthogonal in the multipath optical channel.

The rest of the paper can be summarized as follows: In section 2, we give a comprehensive description of both the VLC and LI-FI system in the indoor work space. Detailed description of multiple access is presented in section 3 . In section 4 , we analyze the simulations of the proposed approach .Finally, some concluding remarks regarding this research.

\section{Theoretical analysis of the system model}

\subsection{Bidirectional in the optical attocell networks}

In view of enhancing the spectral efficiency of the optical communication system, bidirectional communication has been considered to be an efficient way to boost the mobility and easy connectivity of the user, while the connection between several LEDs or APs constitute the networked system. This bidirectional cellular network is known as an optical LI-FI attocell network. Similar to femtocell network, a LIFI cellular consists of several LIFI attocells and provides full functionality given by a cellular system concept, whereas its size is smaller than RF femtocell. The typical geometrics of the optical cellular is shown in Fig. 2 based on the square deployment of the APs. On the downlink side, visible light is used for dual functionality (illumination and communication) provided by optical attocell from the same high-brightness LEDs (AP), while on the other side, uplink is typically accomplished by using IF light with the purpose of avoiding the intra-cell interference and without affecting the illumination restrictions of the room. As illustrated in Fig. 2, the

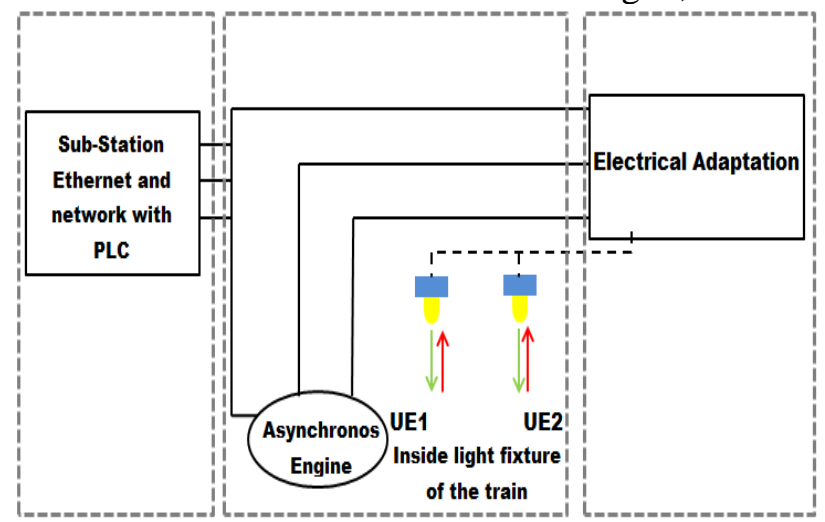

(a)

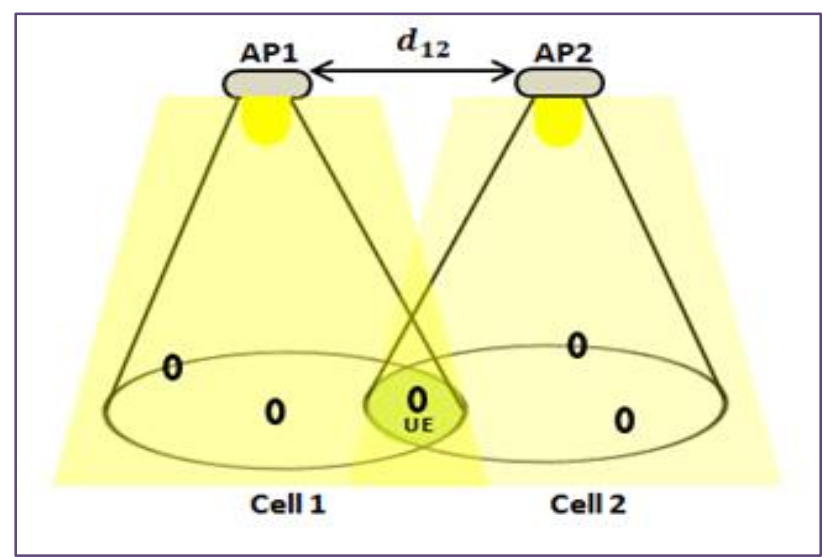

(b)

Figure. 1 Optical wireless communication: (a) proposed LI-FI system in the train and (b) interference in the optical cellular system

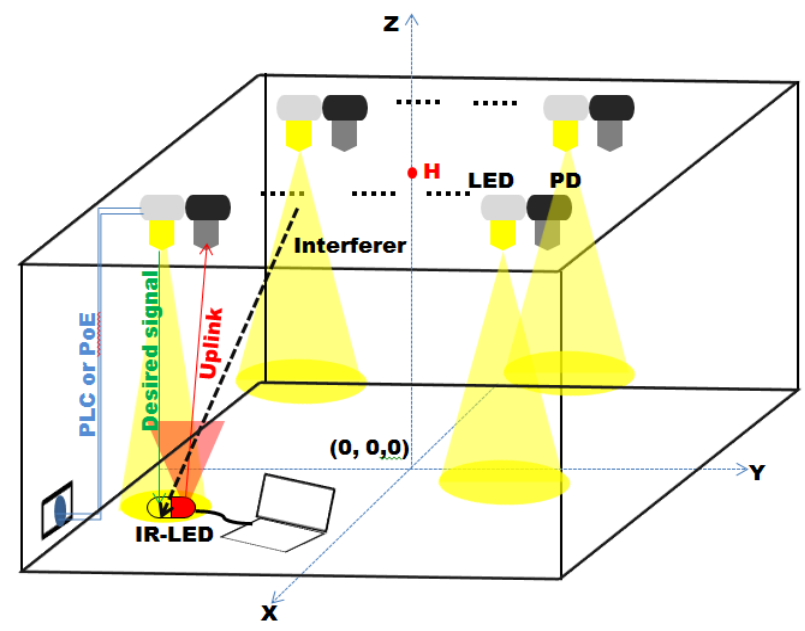

Figure. 2 The setting geometric of an indoor LIFI cellular system

LI-FI network contains multiple APs consisting of a white LED installed on the square lattice ceiling of an indoor space. Next to the LED, a PD is placed as a receiver of the uplink data which is set on the IR frequency band. Also, universal serial bus (USB) 
used by a laptop is faced upward to AP, while IRLED is employed to transmit uplink data and a PD settled on the visible light band is utilized for receiving downlink data. The radiation intensity of the LEDs is supposed to be points source with generalized lambertian emission patterns. Further, the back-haul is realized through using the PLC or power-over-ethernet (POE), where both connections supply the light. A set of assumptions are listed to be considered in this paper before delving into the details of the proposed method. Firstly, the liaison between the APs and users is built upon line-of-sight (LOS) link. Secondly, the positions of the APs are oriented downward and all users are directed upwards. Finally, in fulfilling the light geometry, the light emitted from each AP generates an ideal cone of light while its entire output is presented as a circular lighting area with a hard boundary as shown in Fig. 1 (b). Thus, the coverage area of each cell has a circle form, which is localized at the AP position. It must be noted that the idea behind giving these assumptions in this paper is to put the content of the paper in the tractable path in order to obtain the adequate close-form expression. However, the fundamental concepts of this paper can be used in the places where the fixture lights are installed close to each other like in hospital or library.

\subsection{Indoor geometry of the light propagation model}

To study the efficiency of the optical wireless communication in the attocell network context, a system level evaluation is used to analyze the geometry characteristic of the channel. Besides, the user movement results in higher transmission data rate compared with the channel variation. However, the user movement in indoor circumference is generally static or moving in slow velocity. In this study, users are deemed to be quasi-static which means that the handover is outside the scope of this work. Although this latter won't be considered in this study but a comprehensive overview about the mobility of the user will be discussed without taking it into account in the rest of the simulation results but the purpose is to give a general summary of the system on both sides: static and mobility. In addition, this investigation will be focused only on the downlink performance relying on an achievable SINR as a criterion to evaluate the performance of the proposed method. As detailed in the last section, the design of the optical LIFI attocell is presented in two cases (downlink and uplink), whereas in Fig. 3 the downlink geometry of the optical wireless channel is considered to be the key enabling element in this study. A UE is suggested to be placed at a specific place like a desktop where the laptop is put. It is also equipped with a PD receiver installed on the top of the UE with a field of view (FOV). Furthermore, the angle between the normal of the PD surface with the maximum incident angle that represents the FOV should be sufficiently wide with a view to allowing simultaneous detection of optical signals from the AP. A single LED or an array of the LEDs which creates the AP is assumed to be set up on the ceiling of a typical indoor environment and facing downward to the receiver while each array is usually analyzed as a single point source.

To analyze the effects of channel distortion, a light propagation model is performed to estimate the signal power coming from an assigned AP which is directly related to impulse response variation .Further, two types of propagation model are considered to calculate the path loss, these being LOS nd non-LOS (N-LOS) configuration .In LOS layout, the early knowledge of the transmitter beam divergence , receiver location and separation distance between the $\mathrm{UE}$ and AP are considered to be the chief elements to calculate easily the path loss without taking into account the reflections in the indoor environment. However, non-LOS configuration makes the path loss calculation more complex due to using room reflections, surfaces and furniture. In this study, only the LOS path is regarded as the visible light propagation model and reflections path are outside the scope, in which light is the dominant transmitted signal component used in the downlink indoor environment. This is due to the quantity of the collected energy at the photodiode (PD) which comes from the LOS direction according to [21] The flat frequecy response is considered to be e over the individual subcarrers in the downlink VLC channel 1 which is the acceptable assumption owing to using only the LOS path. Additionally, dividing the total system bandwidth into subcarriers leads to mitigating the channel selectivity in both time and frequency even if the NLOS diffuse homogeneous is used. Before we get into modeling the channel using DC gain, it should be stated that the LED structure depends on the dome shape. As shown in Fig. 4, the lambertian emission is determined by the emitted light coming from the planer LED. However, the maximum intensity $I_{0}$ in lambertian radiation plan is perpendicular to the planar surface, and it decreases with the changing of the viewing angle $\theta$ according to expression Eq. (1).

$$
I(\theta)=I_{0} \cos (\theta)
$$

To get a detailed form of the channel, a fundamental structure is regarded in the OWC system to model the 
channel gain DC of using both underlying components LED and large-area PD as follows:

$$
R_{0}(\theta)=\left\{\begin{array}{c}
p_{t} \frac{m_{1}+1}{2 \pi} \cos ^{m_{k}}(\theta) \theta \in\left[\frac{-\pi}{2}, \frac{\pi}{2}\right] \\
0 \quad \theta \geq \frac{\pi}{2}
\end{array}\right.
$$

Where $m_{k}$ denotes lambertian emission order which expresses the directivity of the source beam by $m_{k}=\frac{-\log (2)}{\log \left(\cos \left(\psi_{1 / 2}\right)\right)}$ and $R_{0}(\theta)$ is the radiation intensity of the LED. As illustrated in Fig. 3, the LOS path between k-th AP and $\delta$-th UE is located at a distance $d$ and angle $\theta$ with respect to the transmitter position which can be written as follows: the radiation intensity

$$
H_{k, \delta}=\frac{A_{p d}(m+1) \cos ^{m_{k}}\left(\theta_{k, \delta}\right) \cos \left(\phi_{k, \delta}\right) g\left(\phi_{k, \delta}\right)}{2 \pi d_{k, \delta}}
$$

Where $A_{p d}$ is the physical area of the detector user, $\psi_{1 / 2}$ is the LED half intensity viewing angle of the AP, $\mathrm{d}_{\mathrm{k}, \delta}$ is the distance between $\mathrm{k}$-th AP and $\delta$-th user or receiver, $\theta_{\mathrm{k}, \delta}$ is the angle of the radiance with respect to the axis normal to the $\mathrm{k}$-th transmitter surface, $\emptyset_{\mathrm{k}, \delta}$ is the angle of incidence with respect to the $\delta$-th receiver surface (user).In Eq. (3), $\mathrm{g}\left(\emptyset_{\mathrm{k}, \delta}\right)$ is the optical concentrator gain of the optical filter which is given as follows :

$$
g\left(\phi_{k, \delta}\right)=\left\{\begin{array}{c}
\frac{\zeta^{2}}{\sin ^{2}\left(\psi_{c}\right)} 0 \leq \phi_{k, \delta} \leq \psi_{c} \\
0 \phi_{k, \delta} \geq \psi_{c}
\end{array}\right.
$$

Where $\psi_{\mathrm{c}}$ and $\varsigma^{2}$ are the receiver FOV and the refractive index. Fig. 3 also shows the analytical geometry rules which can determine the radiance angle $\theta_{\mathrm{k}, \delta}$, the incidence angle and the $\delta$-th UE as follows:

$$
\begin{aligned}
& \cos \left(\theta_{k, \delta}\right)=\frac{\overrightarrow{d_{k, \delta}} \cdot \overrightarrow{n_{T X}}}{\left\|d_{k, \delta}\right\|} \\
& \cos \left(\phi_{k, \delta}\right)=\frac{\overrightarrow{d_{k, \delta}} \cdot \overrightarrow{n_{R X}}}{\left\|d_{k, \delta}\right\|}
\end{aligned}
$$

$n_{T x}=[0,0,-1]$ and $n_{R x}=[0,0,1]$ are the normal vectors at the transmitter and $\delta$-th at the receiver planes ,respectively. "." and || . || signify the inner product and the Euclidean norm operators, respectively. By replacing $\cos \left(\theta_{k, \delta}\right)=\cos \left(\emptyset_{k, \delta}\right)=\frac{h}{d_{k, \delta}}$ and normal vectors, $\mathrm{H}_{\mathrm{k}, \delta}$ can be rewritten as function of $\mathrm{r}_{\mathrm{k}, \delta}$ and $d_{k, \delta}=\sqrt{r_{k, \delta}{ }^{2}+h_{k}^{2}}$, where $\mathrm{r}_{\mathrm{k}, \delta}$ refers to the horizontal separation between the UE and k-th AP. Towards this end, the LOS path model $\mathrm{H}_{\mathrm{k}, \delta}$ can be converted to a function of $r_{k, \delta}$ as follows:

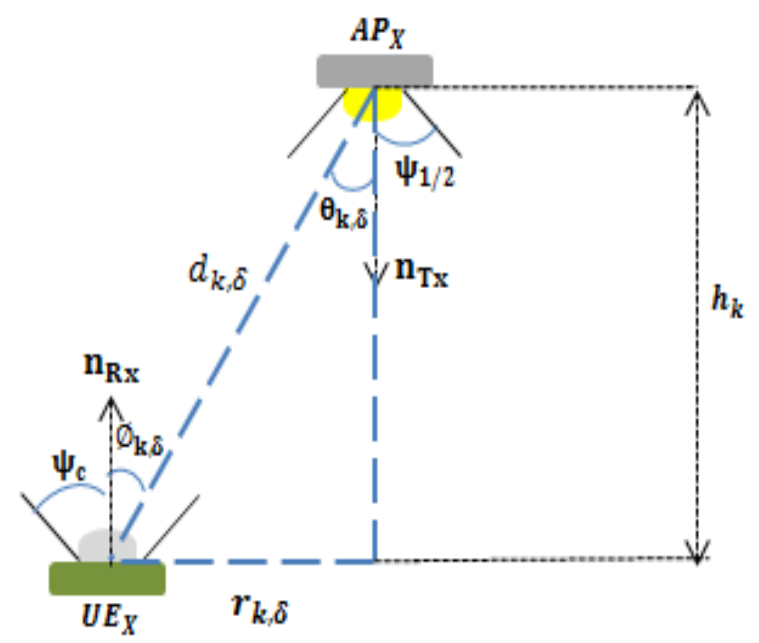

Figure. 3 Two -dimensional (2D) -LOS light propagation geometry in optical attocell system

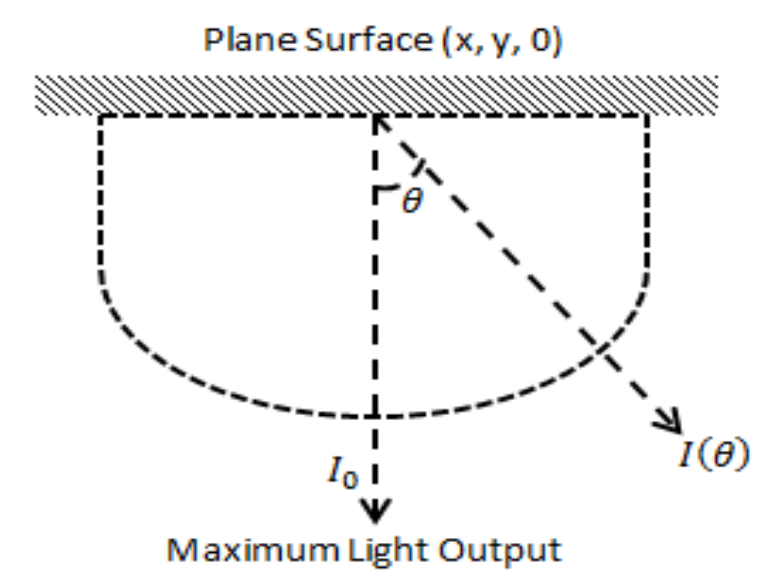

Figure. 4 The basic structure of the LED

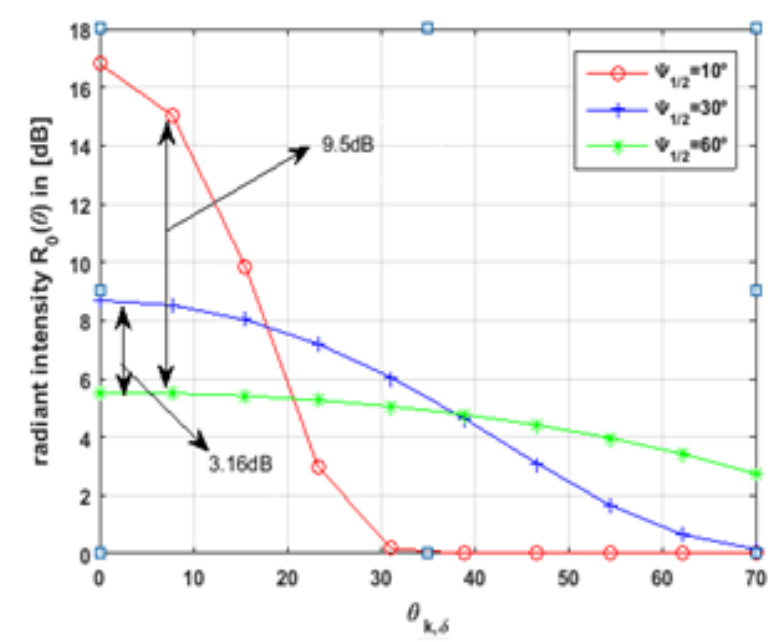

Figure. 5 The radiant intensity of the transmitter 


$$
H_{k, \delta}=\frac{A_{p d}(m+1) h^{m_{k}+1} g\left(\phi_{k, \delta}\right)}{2 \pi\left(r^{2}{ }_{k, \delta}+h_{k}\right)^{\frac{m_{k}+2}{2}}}
$$

Note that $h_{k}$ is the normal distance between the AP and the UE planes. The optical concentrator gain usually takes the value $1 g\left(\phi_{k, \delta}\right)=1$ if lens isn't used at the receiver. The large area of the detector $\left(A_{p d}\right)$ leads to collecting an important quantum of the light and thus increases its sensitivity. Therefore, there is a tradeoff between sensitivity and bandwidth which makes the area of the detector to be chosen not too large. The influence of different sizes of PD on systems performances is shown in [22]. In addition, the performance of a downlink-VLC system depends on many factors as given by Eq. (3). Predefined system elements related to the network configuration control some parameters of the light propagation model. One of the parameters is the LED half intensity viewing angle. The other parameter is the angle of the AP radiance .As shown in Fig. $5, \mathrm{R}_{0}(\theta$ is controlled by calculating three different values of the LED half intensity according to Eq. (2) in order to illustrate how this latter influences the radiation intensity. By moving the angle of the radiance $\theta_{\mathrm{k}, \delta}$ which represents the displacement of the UE according to the plane $\left(X_{n}, Y_{n}\right)$ and based on the assumption $\theta_{k, \delta}=\emptyset_{k, \delta}$, the UE with $\psi_{1 / 2}=60^{\circ}$ shows the good performance even if its maximum intensity is $5 \mathrm{~dB}$, whereas both UEs with $\psi_{1 / 2}=30^{\circ}$ and $\psi_{1 / 2}=10^{\circ}$ converge to 0 at the $\theta_{k, \delta}=30^{\circ}$ and $\theta_{k, \delta}=70^{\circ}$.Therefore, the Lambertian orders is $\mathrm{m}=1$.

\subsection{The low pass characteristics of the Front-End Device-LED}

A comprehensive analysis has been conducted to mitigate the effect of the nonlinearity coming from the LED [23, 24]. However, some investigations have considered the LED as a linear component in spite of the fact that a set of the fundamental aspects of the LED modulation behavior cannot be deducted from the linear model. In this study we will focus on the response frequency of the LED which influences both the total channel gain $H(K)$ and free space transmission where the former is modeled by $\mathrm{H}_{\mathrm{k}, \delta}$. Consequently, $\mathrm{H}(\mathrm{K})$ can be written as follows:

$$
|H(k)|^{2}=\left|H_{L E D}(k)\right|^{2}\left|H_{k, \delta}(k)\right|^{2}
$$

Where $H_{L E D}(K)$ is the frequency response of the LED and $\mathrm{H}_{\mathrm{k}, \delta}(K)$ represents the frequency response of the channel gain in the free-space transmission. The filtering effect of the LED will be subsequently discussed in this part. In order to better understand the general effect of the LED, a detailed explanation about the low pass characteristics of LED are illustrated in Fig.
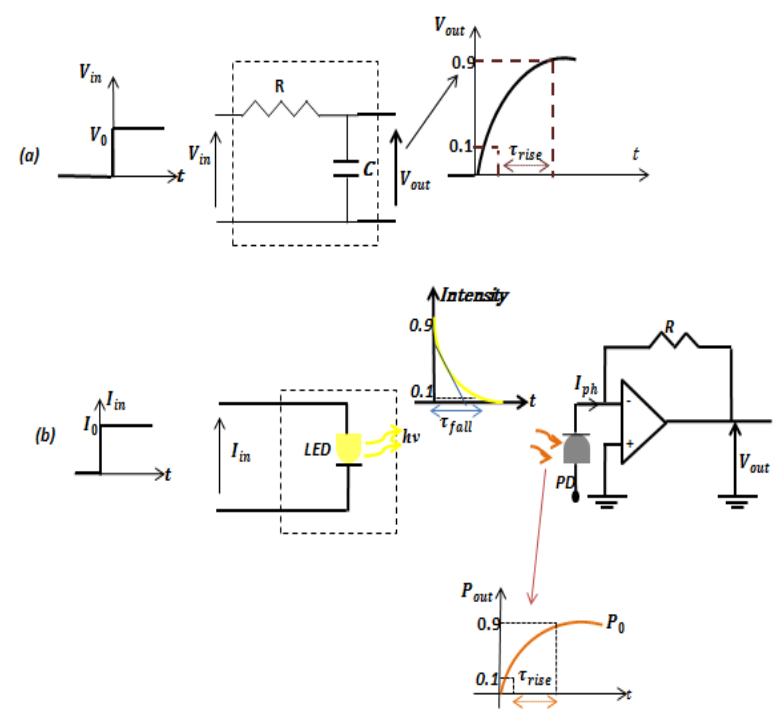

6, whereas a sample RC circuit is used to explain the

Figure. 6 The system response of the linear RC circuit and LED

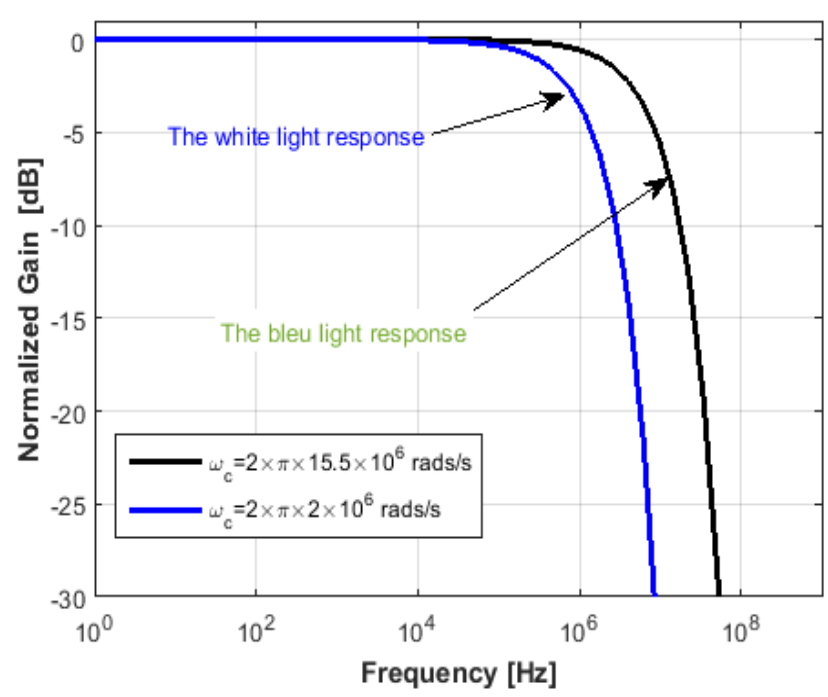

Figure. 7 The modulation bandwidth of the LED

general effect of the filtering as shown in Fig. 6 (a). A step -function input voltage $V_{\text {in }}$ is subject to the effect of the $\mathrm{RC}$ circuit in order to evaluate its performance on the output voltage $V_{\text {out }}$. Therefore, the characteristics of the LED can be modeled as the low pass filter shown in Fig. 6 (b), while a step function input current is used in the LED's input. The photocurrent $\mathrm{I}_{\mathrm{ph}}$ is generated by the light falling on the area of the PD in order to calculate the light output power $\mathrm{P}_{\text {out }}$ as function of time. In Addition, the 3-dB bandwidth of the LED is typically low due to the $\tau_{r}$ rise and full time $\tau_{\mathrm{f}}$ of the capacitance and resistance 
of the LED. The frequency response of the LED can be modeled as follows

$$
H(\omega)_{L E D}=e^{-\left(w / w_{c}\right)}
$$

Fig. 7 shows the theoretical frequency response of the LED with two different fitted coefficients $\left(\mathrm{w}_{\mathrm{c}}=\right.$ $2 \pi \times 15.5 \times 10^{6} \mathrm{rad} / \mathrm{s}$ and $\mathrm{w}_{\mathrm{c}}=2 \pi \times 2 \times$ $10^{6} \mathrm{rad} / \mathrm{s}$ ), whereas the frequency response of the blue LED presents large bandwidth (15Mhz) compared with frequency response of the white LED (2Mhz) [25].

\subsection{The hexagonal cellular of two optical attocell network with polar coordination}

In the work environment, a simplified model is used to evaluate the UE performance by relying on the two-layer hexagonal network deployment due to the location of the UE at the edge of the coverage area [26]. In addition, the cell circle radius of the LED and radius of the hexagonal cell play a vital role in determining the signal strength distribution within the coverage area of the cell and minimizing the CCI effect according to the cell size. Besides, prioritizing the use of the HEX deployment rather than using the PPP is included in the concept of the coordinate's location of the AP. The coordinates of all neighboring interfering APs are well known for the desired user compared to the PPP cell deployment where the coordinates of the AP are randomly placed according to the absence probability of the user in the cell. In the FFR system both cases $\Delta=1$ and $\Delta=3$ are employed to explain the location of the user and APs. In Fig. 8, a geometric model is shown to simplify the explanation of the coverage area of the cell. Furthermore, this latter is divided into a cell center area and cell edge area with a circular cell approach that represents the central cell. The circular cell is determined as $\mathrm{r}_{\mathrm{e}} \approx 0.91 \mathrm{R}$ owing to the approximation between the circular cell and the original hexagonal cell. The radius of the central area is defined as $r_{c}$. The size of the cell central and edge area is determined by the appropriate setting of the parameter $\delta=\frac{r_{c}}{r_{e}}$. Moreover, the location of the user and interfering AP is one of the most key enabling elements in determining the service quality level by calculating the achievable SINR and using two dimensional polar coordinations shown in Fig. 8. The origin of the coordinates is set at the location of $\mathrm{AP}_{0}$ by taking into account the orientation of the polar axis. $l=$ $(r, \theta)$ determines the location of the user in the central cell, where $r$ is the horizontal distance between the user and the origin $r \in\left[0, r_{e}\right]$ and $\theta$ is the polar angle of the user $\theta \in[0,2 \pi)$. Likewise, the coordinate $\left(\mathrm{R}_{\mathrm{k}}, \Theta_{\mathrm{k}}\right)$ defines the location of the k-th AP, where $R_{k}$ is the horizontal distance between $\mathrm{AP}_{\mathrm{k}}$ and the origin, and $\Theta_{\mathrm{k}}$ is the polar angle of $\mathrm{AP}_{\mathrm{k}}$. The fixed values of the $\left(R_{k}, \Theta_{k}\right)$ can be easily calculated due to the availability of two factors: the network deployment and the cell radius $\mathrm{R} . \mathrm{r}_{\mathrm{k}}(l)$ which is the horizontal distance between

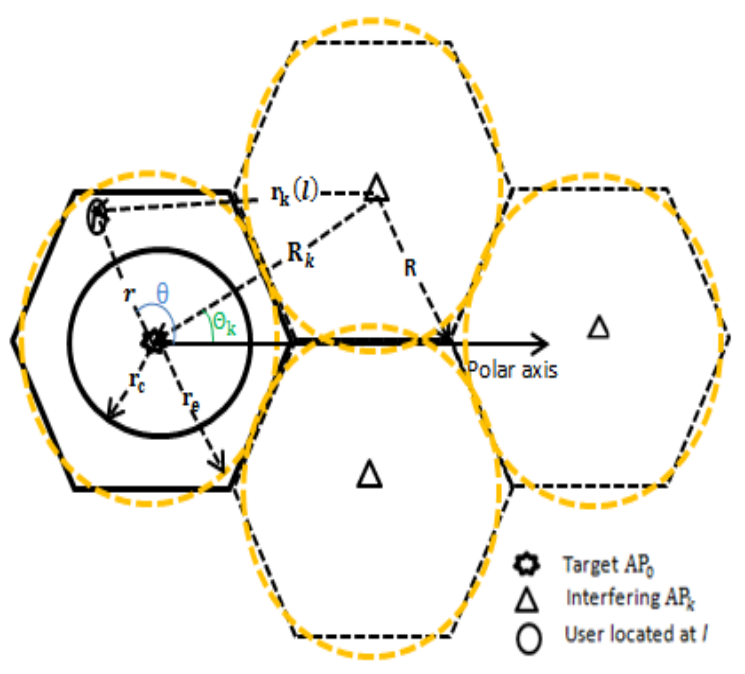

Figure. 8 The 2D downlink model with the polar axis

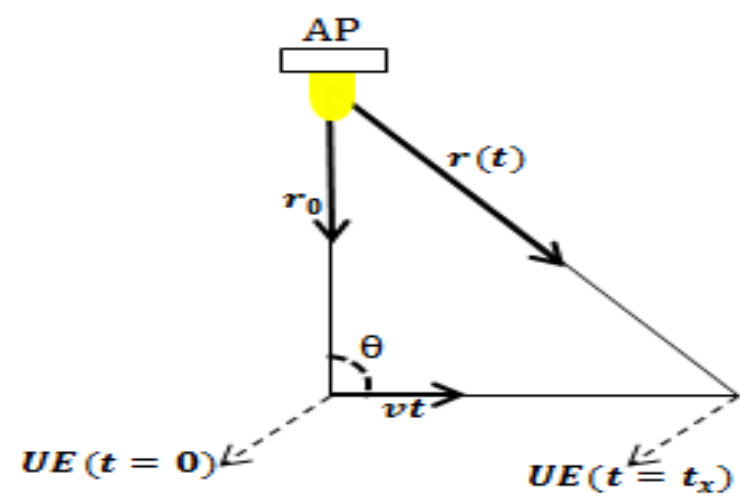

Figure. 9 The random waypoint model (RWP)

the $\mathrm{AP}_{\mathrm{k}}$ and the user at the $l$ plays an important role in determining the signal power coming from the $\mathrm{AP}_{\mathrm{k}}$ and directed to the desired user. For $k=0, r_{0}(l)=r$.From the geometry of the cell deployment, the $\mathrm{r}_{\mathrm{k}}(l)$ can be defined as follows:

$$
r_{k}(l)=\left(r^{2}+R_{k}^{2}-2 R_{k} r \cos \left(\theta-\Theta_{k}\right)^{\frac{1}{2}}\right)(10)
$$

It is worth noting that the user mobility sample can be modeled by the random waypoint model (RWP) which is commonly used in the wireless communication network [27].In addition, reliance on three fundamental rules, namely: random distinction (uniformly chosen by probability $1 / \pi \mathrm{r}_{\mathrm{e}}{ }^{2}$ ), movement path and user velocity, lead to determining the UE's movement from waypoint to another waypoint as shown in Fig. 9. In this case, the distance between the $\mathrm{AP}$ and UE at time instance $\mathrm{t}$ is $d(t)=\sqrt{r^{2}(t)+h^{2}}$, 
where, $r(t)=\sqrt{\left(r^{2}{ }_{0}+v^{2} t^{2}-2 r_{0} v t \cos (\theta)\right)}$ with $\theta=\pi-\cos ^{-1}\left(\frac{\overrightarrow{r_{0}} \cdot \vec{v}}{\left|\overrightarrow{r_{0}}\right||\vec{v}|}\right) ; \overrightarrow{r_{0}}$ is the initial distance vector from the cell center at $\mathrm{t}=0$ with $\left|\overrightarrow{r_{0}}\right|=r_{0}$ and $\vec{v}$ is the velocity vector of the UE with $\left|\overrightarrow{v_{0}}\right|=v_{0}$

\section{Multiple access and downlink throughput calculation}

In view of data transmission with visible light which is achieved through the IM/DD system, many typical modulation techniques can be employed in the OWC system. Therefore, single-carrier pulse modulation is an adequate element for data transmission based on data encoding in the duration of the pulse like pulse width modulation (PWM) [28]. The pulse position modulation (PPM) is one of the orthogonal modulations where the information is encoded within the duration of the pulse [29]. Additionally, the data can be encoded in the amplitude of the pulse during each symbol interval such as pulse amplitude modulation (PAM) [30]. A flurry of attempts with single-carrier modulation seeks to increase the throughput in a dispersive channel due to surpass of the pulse bandwidth in relation to the coherence channel. This last phenomenon leads to unwanted effects like intersymbol interference (ISI) and clipping noise resulting from the LED. In order to remedy these problems facing the single-carrier modulation, equalization has been used to estimate the data as accurately as possible at the receiver side. On the contrary, the multicarrier modulation such as quadrature amplitude modulation (M-QAM) or OFDM are two of the most suitable candidates to combat the ISI and offer higher data rate owing to the longer duration of the symbol than the root mean square (RMS) of the optical wireless channel. Even more, OFDM has been primordially considered in the downlink-VLC system according to its strict performance such as the inherent forcefulness against frequency selectivity of channel by dividing the channel into narrowband flat fading subcarriers, the effective management of the spectrum, and finally the multiplexing and demultiplexing which are used at both sides transmitter and receiver through using the fast fourier transform (FFT) and its inverse (IFFT) [31]. It should be pointed out that the time- domain signal must be positive and real-valued for the purpose of modulating the LED intensity. DCO-OFDM is one of the very well-known modulation schemes that can be adopted in the downlink VLC system. Fig. 10 shows the signal processing diagram of the downlink DCOOFDM and its main blocks. For each OFDM frame, the information bits are firstly mapped to QAM symbols. Afterwards, the serial-to-parallel converter is applied on the modulated symbols stream which is grouped and converted to parallel frames of OFDM and fed into the input of the IFFT block. An OFDM symbol can be introduced as follows:

$$
X=\left[X_{0}, X_{1} \cdots \cdots X_{N-1}\right]
$$

Where $\mathrm{X}_{\mathrm{p}}$ for $\mathrm{p}=0, \ldots \mathrm{N}-1$ are modulated data symbols which are transmitted on $\mathrm{f}_{\mathrm{p}}$-th OFDM subcarriers given by $f_{P}=P \Delta_{f}$ with $\Delta_{\mathrm{f}}$ is the frequency spacing between two adjacent subcarriers, where $\mathrm{N}$ is the number of the subcarriers .The OFDM symbols are subjected to Hermitian symmetry operation for ensuring the real value signals in the time domain. Therefore the OFDM symbols are normalized by a factor of $\xi=\frac{N}{N-2}$. After the IFFT operation, time domain samples are expressed as follows:

$$
\begin{gathered}
x[n]=\frac{1}{\sqrt{N}} \sum_{p=0}^{N-1} X_{p} \exp \left(\frac{j 2 \pi p_{n}}{N}\right), \\
0 \leq n \leq N-1
\end{gathered}
$$

Where $\mathrm{x}[\mathrm{n}]$ follows a Gaussian distribution with zero mean and unity variance. After the IFFT processing block, a cyclic prefix (CP) will be added to the samples by regarding the length of the maximum delay of the channel. In addition, $\mathrm{N}$ transformed data symbols then pass through parallelto-serial converter which will be fed into a digital to-analogue converter (DAC) module. To confirm that the modulated signal $\overline{x(t)}$ is positive, a DC bias is added to the analogue waveform in order to modulate the intensity of the light. Therefore $\mathrm{x}_{1}(\mathrm{t})$ is given as follows:

$$
x_{l}(t)=x_{D C}+\overline{x(t)}
$$

Where $x_{D C}=\gamma \sqrt{E\left[x^{2}(t)\right]}$ and $\gamma$ is the conversion factor. The clipping noise is negligible due to the condition $\gamma=3$ that ensures that less than $1 \%$ of the signal is clipped [8]. The optical power is obtained by the signal $x_{1}(t)$ that drives the LED. As we have seen earlier about the ISI effect that is solved by the subcarriers orthogonality and cyclic prefix, ICI is still the main problem in the cellular system which results by using the same subcarriers in neighboring APs at the same time. Dividing the available subcarriers into a set of subchannels allows the users in the cellule to be stronger against collision and mitigates the ICI effects. Therefore, the main idea is to assign time- 
frequency resource to a group of users in the same time which results in a new multiple access named
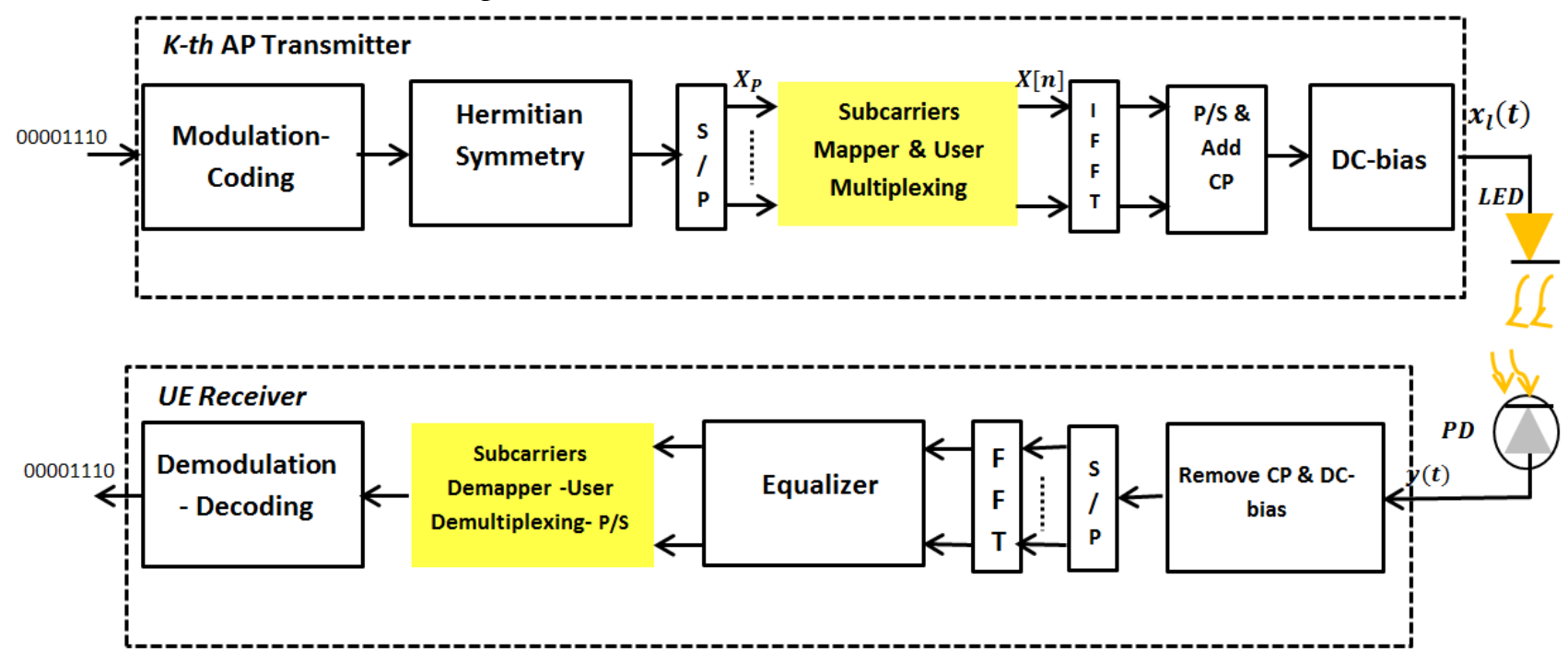

Figure. 10 DCO-OFDMA downlink system

OFDMA. The yellow block in Fig. 10 shows the subcarrier mapper that allocates users' data symbols to subcarriers, while the allocation of the resources is controlled in both the time domain (slots) and the frequency domain (tone). OFDMA considered in this study builds upon the using of the multiple optical APs. These APs have the responsibility to cover the network by their signals power in order to serve the desired users. However, the reaming of the APs that uses the same resources produces CCI effect to the desired users. As mentioned above, a subscript $\mathrm{k}$-th $=0,1$, $2 \ldots$ is used to identify the signal coming from different APs. The intended AP corresponds to the case of $\mathrm{k}=0$, where $\mathrm{k} \in \Pi$ corresponds to the case of the interfering APs, in which $\Pi$ denotes the group of the APs using the same resources. Therefore, the received signal of the desired user $\delta$ at time slot t can be expressed as follows:

$$
\begin{array}{r}
y(t)=\lambda_{p d}\left[x_{0}(t) \otimes h_{0}(t)+\sum_{k \in \Pi} x_{k}(t) \otimes\right. \\
\left.h_{k}(t)+n_{R X}(t)\right]
\end{array}
$$

Where $\lambda_{p d}$ is the PD responsivity of the receiver, $h_{k}(t)$ is the channel impulse response in the time domain of the downlink VLC system from the k-th AP to the desired user and $n_{R X}(t)$ represents the time domain noise at the receiver. After the two processing blocks: the FFT operation and the single tap equalization shown in Fig. 10 at the receiver side, the original QAM symbols can be recovered. According to Eq. (3), the signal-noise-ratio (SNR) of the $\delta$-th UE on $\mathrm{p}$-th subcarrier can be obtained as follows:

$$
\begin{aligned}
S N R_{D, \delta} & =\frac{N \lambda_{p d}{ }^{2} P_{D}^{2} H_{k, \delta}{ }^{2} H(\omega)_{L E D}{ }^{2}}{(N-2) \gamma^{2} N_{0} B_{D}} \\
& =\frac{M e^{\left(\frac{-4 \pi B_{D}}{N \omega_{c}}\right)}}{\left(r_{k, \delta}{ }^{2}+h_{k}^{2}\right)^{m+3}}
\end{aligned}
$$

Where

$M=\frac{N \lambda_{p d}{ }^{2}\left(A_{p d}(m+1) h^{m_{k}+1} g\left(\emptyset_{k, \delta}\right)\right)^{2} P_{D}{ }^{2}}{(2 \pi)^{2}(N-2) \gamma^{2} N_{0} B_{D}}, \mathrm{P}_{\mathrm{D}}$ is the transmitted optical power by each $\mathrm{AP}, \mathrm{N}_{0}$ is the noise power spectral density and $B_{d}$ is the downlink reused bandwidth due to FFR and $w=p B_{D} / N$.After that , the throughput of $\delta$-th UE can be expressed as follows:

$$
R_{D, \delta}=\frac{B_{D}}{N} \sum_{p=1}^{N / 2-1} \log _{2}\left(1+S N R_{D, \delta}\right)
$$

Substituting (15) by (16), the downlink throughput is rewritten as follows:

$$
R_{D, \delta}=\frac{B_{D}}{N} \sum_{p=1}^{N / 2-1} \log _{2}\left(1+\frac{M e^{\frac{-4 \pi p B_{D}}{N w_{c}}}}{\left(r_{k, \delta}{ }^{2}+h_{k}^{2}\right)^{m+3}}\right)(17)
$$

In the communication system, SINR is considered to be an essential metric tool to evaluate the network quality and the transmission throughput while it is defined as the ratio of the received desired signal power to the total noise and interference power. The received SINR of $\delta$-th UE on p-th subcarrier can be written as follows:

$$
\operatorname{SINR}_{D, \delta}
$$




$$
=\frac{\lambda_{p d}{ }^{2} P_{D}{ }^{2} H_{k, \delta}{ }^{2} H(\omega)_{L E D}{ }^{2}}{(N-2) \gamma^{2} \sigma_{\delta}{ }^{2}+\sum_{k \in \Pi, k \neq 0} \lambda_{p d}{ }^{2} P_{D}{ }^{2} H_{k, \delta}{ }^{2} H(\omega)_{L E D}{ }^{2}}
$$

Where $\sigma_{\delta}^{2}$ is the noise on $\mathrm{p}$-th subcarrier of $\mathrm{UE}_{\delta}$. According to the user location $l$ and Eq. (10) on pth subcarrier, the SINR can be rewritten as follows :

$$
\operatorname{SINR}_{D, \delta}=\frac{\left(r_{k, \delta}{ }^{2}+h_{k}^{2}\right)^{-m-3}}{\sum_{k \in \Pi, k \neq 0}\left(r_{k}^{2}(l)+h_{k}^{2}\right)^{-m-3}+\beta}
$$

Where, $\beta=\frac{4 \pi^{2}(\mathrm{~N}-2) \gamma^{2} N_{0} B_{D}}{N \lambda_{p d}{ }^{2} \mathrm{~A}_{\mathrm{pd}}{ }^{2}(\mathrm{~m}+1)^{2} \mathrm{~h}^{2 \mathrm{~m}_{\mathrm{k}}+2} \mathrm{~g}\left(\emptyset_{\mathrm{k}, \delta}\right)^{2} P_{D}{ }^{2}}$

\section{Analysis of the proposed model and simulation results}

\subsection{The geometry design of the proposed resource allocation}

For the sake of simplicity, we first consider an indoor communication downlink scenario which is made up of two APs and three UEs, while one of the APs being the interfering cell as shown in Fig. 11. OFDMA modulation scheme is proposed to be used in the coverage area of the AP for the reason of serving multiple users. As illustrated again in Fig. 11, we adopt the FFR $\Delta=3$ pattern to reduce interference and increase SINR for UE near the edge of the coverage area.Therefore, we partition the coverage area of each AP into three regions, namely region 1 , region 2 and region 3 , where the users located in each region are indicated as inferior users in region 1 , middle users in region 2 and edge users in region 3.After defining the three categories of the users, they are considered to be uniformly distributed in different regions. Regarding the assumptions shown above in Fig. 2, three UEs are assumed to be distributed in different regions. We suggest that the UE will use infrared in order to provide AP the required information like the strength of the received signal, SINR values, and the knowledge about the UE location. In addition, the radius $r_{1, k}[m], r_{2, k}[m]$ and $r_{3, k}[m]$ are related to the different regions in the k-th AP. Hence, the total radius of the coverage area is $r_{k}=r_{1, k}+r_{2, k}+$ $r_{3, k}[m]$.However, it should be pointed out that when no limitations are regarded, the radius of the region 1 is $r_{1, k} \leq r_{k}$ with respect to $r_{3, k} \geq r_{2, k} \gg$ 0 .It is worth mentioning that the subcarriers are shared among the UEs in the different regions and at each AP owing to dividing the available bandwidth. In doing so, we determine a set of assumptions after getting into details. Let us consider $\mathrm{P}_{\mathrm{c}, \mathrm{k}}, \mathrm{B}_{\mathrm{c}, \mathrm{k}}$ and $\mathrm{N}_{\mathrm{c}, \mathrm{k}}$ respectively as the total optical power, the total available bandwidth and the total number of subcarriers in the cell. In the different regions of the served UE, the corresponding allocated resources are referred to as $\mathrm{P}_{\mathrm{R}, \mathrm{k}}, \mathrm{B}_{\mathrm{R}, \mathrm{k}}$ and $\mathrm{N}_{\mathrm{R}, \mathrm{k}}$ for $R=\{1,2,3\}$ while the data transmission is made by using all cell resources as follows: $\sum_{i=1}^{R_{T}} P_{i, k}=P_{c, k} ; \sum_{i=1}^{R_{T}} B_{i, k}=B_{c, k} ; \quad \sum_{i=1}^{R_{T}} N_{i, k}=$ $N_{c, k}$, where $\mathrm{R}_{\mathrm{T}}$ is the total region of the coverage area and $U E=\bigcup_{i=1}^{R_{T}} U E_{i}$. Furthermore, to adopt an objective analysis, we restrict it by assuming that the same optical power, bandwidth, and number of subcarriers are used in all APs: $P_{c, k}=P_{c}, \mathrm{~B}_{\mathrm{c}, \mathrm{k}}=$ $\mathrm{B}_{\mathrm{c}}$ and $N_{c, k}=N_{c}$. The features $\mathrm{B}_{\mathrm{p}, \mathrm{k}}$ and $\mathrm{P}_{\mathrm{p}, \mathrm{k}}$ are assumed to be the allocated bandwidth and power at the $\mathrm{p}$-th subcarrier and $\mathrm{k}$-th $\mathrm{AP}$ whereas $B_{p, k}=$ $B_{c} / N_{c}$ and $P_{p, k}=P_{c} / N_{c}$ with respect to $\mathrm{k}=1 \ldots \mathrm{K}$ and $\mathrm{p}=1 \ldots N_{c}$.Subsequently, both elements $\mathrm{r}_{\mathrm{R}, \mathrm{k}}$ and $\mathrm{N}_{\mathrm{R}, \mathrm{k}}$ can characterize each region in the coverage area based on the assumption that $P_{R, k}=N_{R, k} P_{c} / N_{c}$ and $B_{R, k}=N_{R, k} B_{c} / N_{c}$ for $R=\{1,2,3\}$. Fig. 12 shows the assignment of the subcarriers among the users to be served in each region while the available bandwidth is divided into subchannels which constitute of a set of subcarriers to be allocated to each user.

As we have seen earlier, the new optimization that determines how to allocate both the power and subcarrier geometrically based on the equality policy between the mentioned elements. Also, another parameter is going to be optimized in order to enhance the per -user throughput. For the sake of clarity, we assume that the ICI is mitigated between cells, and then reduced to SNR, thus solving the derivation of Eq. (17) as follows:

$$
\begin{aligned}
& R_{D, \delta}=\frac{B_{D}}{N} \sum_{p=1}^{\frac{N}{2}-1} \log _{2}\left(1+\frac{M e^{\frac{-4 \pi p B_{D}}{N \omega_{c}}}}{\left(r_{k, \delta}{ }^{2}+h_{k}{ }^{2}\right)^{m+3}}\right) \\
& =\frac{B_{D}}{N} \sum_{p=1}^{\frac{N}{2}-1} \frac{\ln \left(1+\frac{M e^{\frac{-4 \pi p B_{D}}{N \omega_{c}}}}{\left(r_{k, \delta}{ }^{2}+h_{k}^{2}\right)^{m+3}}\right)}{\ln (2)} \\
& \begin{array}{c}
\Rightarrow \frac{\partial R_{D, \delta}}{\partial r_{k, \delta}}=-\frac{2 B_{D} r_{k, \delta}(m+3)\left(r_{k, \delta}{ }^{2}+h_{k}{ }^{2}\right)^{-m-4}}{N \ln (2)} \\
\sum_{p=1}^{N / 2-1} \frac{e^{\frac{-4 \pi p B_{D}}{N \omega_{c}}}}{1+e^{\frac{-4 \pi p B_{D}}{N \omega_{c}}}}<0
\end{array}
\end{aligned}
$$

Eq. (20) implies that the $\mathrm{R}_{\mathrm{D}, \delta}$ is monotonically decreased with respect to $r_{k, \delta}$ as depicted in Fig.13 .This means that higher throughput can be achieved when the user is being located in region 1 


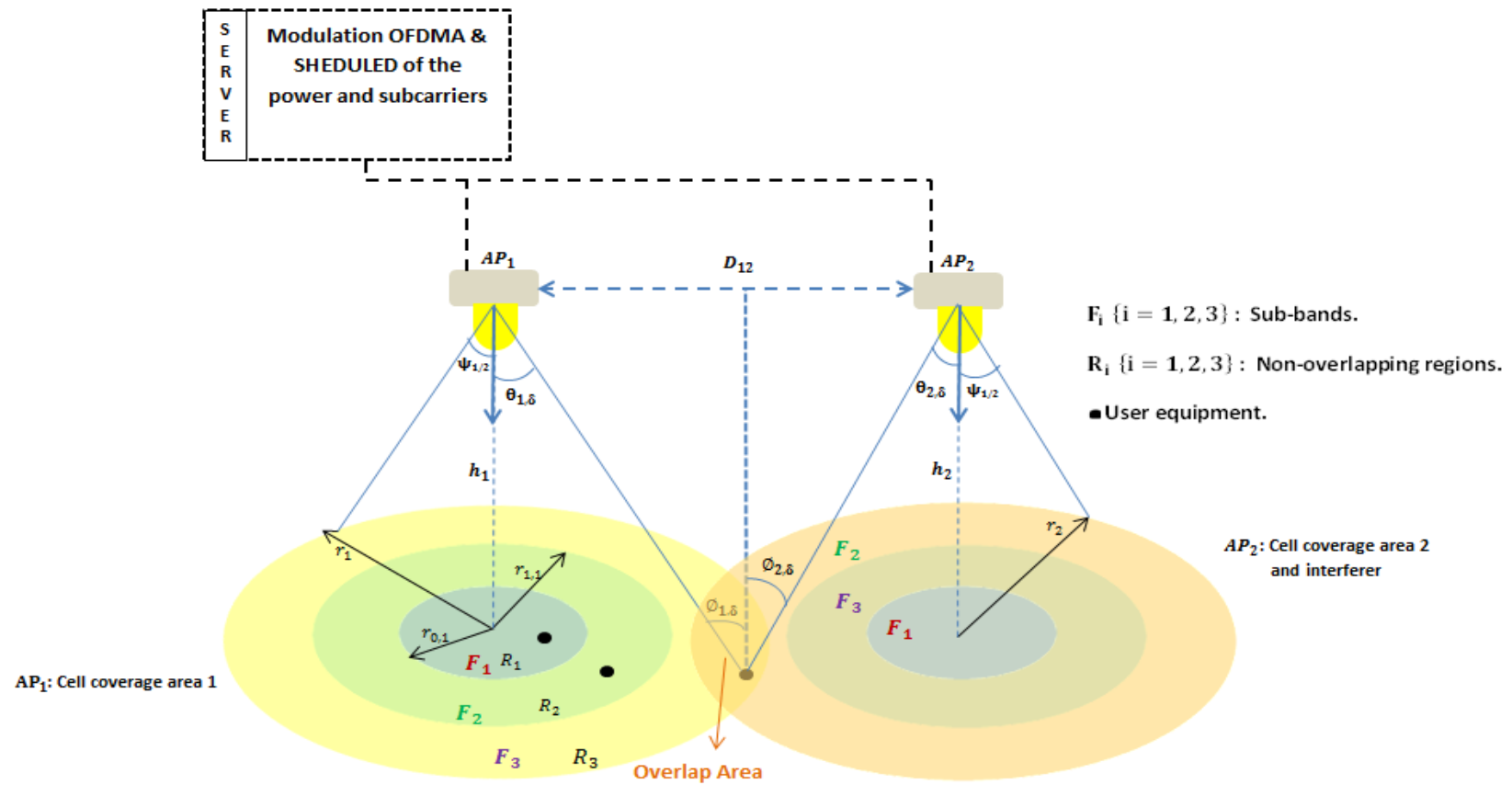

Figure. 11 Geometry model of the proposed resource allocation

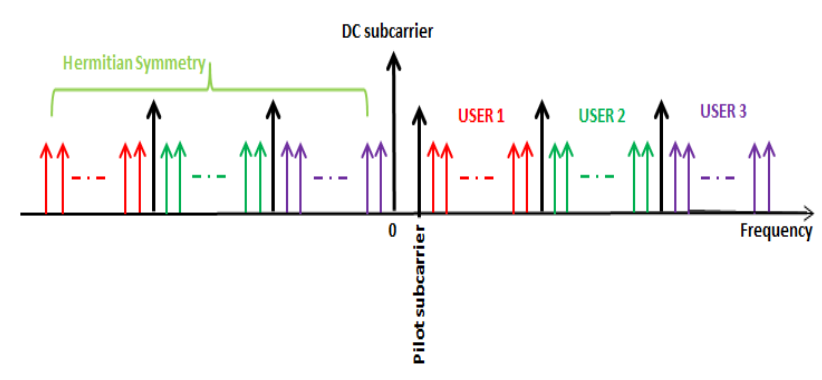

Figure. 12 Subcarriers distribution between users in the downlink optical attocell

$\left(0 \leq r_{1,1} \leq 1 m\right)$, whereas this throughput is reduced in region $3\left(2 m \leq r_{3,1} \leq 3 m\right)$ due to both the large cell and the weakest received signal from the $\mathrm{AP}_{1}$ where $\psi_{1 / 2}=60^{\circ}$. On the other hand, we also notice that $\psi_{1 / 2}=30^{\circ}$ shows a good performance at the cell center compared to the other half intensity, but it reduces the throughput to almost $40 \%$ at the cell edge (region 3) .Depending on the last comparison depicted in Fig. 13, the value $\psi_{1 / 2}=60^{\circ}$ will be utilized to model half intensity in the rest of the paper. In addition the first goal can be directed toward the maximization of the received signal power at the cell edge according to this relation:

$$
E=J_{0} \frac{h^{m_{k}+1}}{\left(r_{k, \delta}{ }^{2}+h_{k}^{2}\right)^{\frac{m_{k}+1}{2}}}
$$

Where $\mathrm{J}_{0}$ is the maximum luminous intensity [cd], $\mathrm{E}[\mathrm{Lx}]$ is the Illumination Level or brightness which characterizes the illuminated surface depending on the European lighting standard [27]. Fig. 14 presents

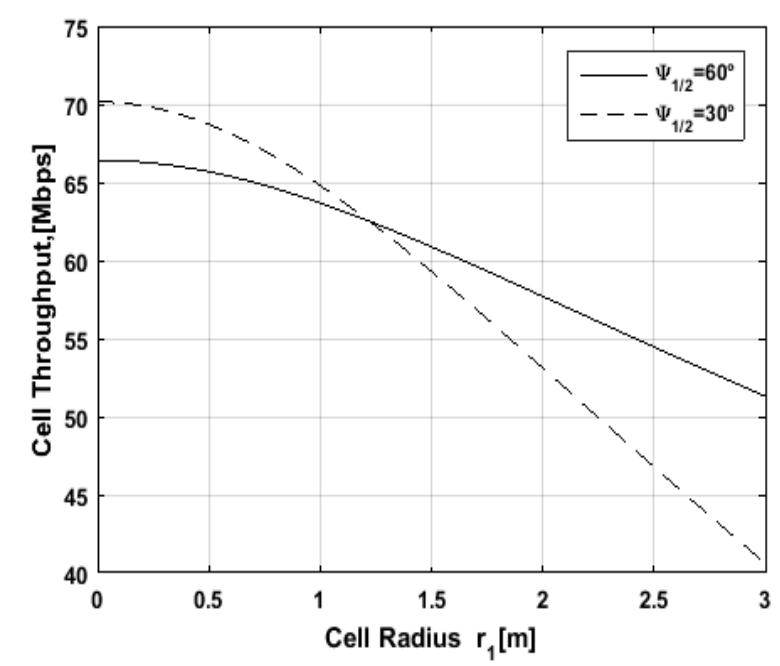

Figure. 13 Overall throughput versus the radius of the coverage area $\mathrm{AP}_{1}$

the luminous intensity evolution at a given location by relying on the minimum and the maximum brightness level which means that a higher intensity is assigned to the users at the cell edge while less brightness will cover the other regions (R2 and R1) of the coverage area.This is caused by solving the derivation of $\frac{\partial \mathrm{P}_{\mathrm{D}}}{\partial \mathrm{m}_{\mathrm{k}}}$ in order to achieve the optimal radiation .We should minimize the strength signal variation among the different regions of the AP in order to guarantee a sufficient amount of light that illuminates the coverage area. As aforementioned in Fig. 5, the optimum value is chosen to be $m=1$ that shows better results on the illumination side compared with the other values . In this case, another 
parameter will be optimized in order to jointly keep the optimum illumination of the coverage area and to enhance the user throughput at cell edge which is our main goal.

With regard to our chief goal which attempts to enhance the throughput of the edge UE under the optimization operation, since it is suggested that the same request throughput is assumed to be demanded by the UEs .In this study, we calculate the average downlink throughput $\mathrm{R}_{\mathrm{D}, \delta}^{\text {avg }}$ over the minimum and the maximum radius of the coverage area under the assumption that the interference can be omitted due to FFR plan. Hence the optimization solution can be formulated as follows:

$$
\max _{\rho_{0}} R_{D, \delta}=\frac{1}{N} \sum_{p=0}^{p_{\min }} R_{D, \delta}\left(\rho_{0}\right)^{a v g}
$$

Where $\rho_{0}$ is the optimization parameter that will be explained below and $p_{\min }$ is minimum subcarriers to satisfy the requested throughput. $\mathrm{R}_{\text {req }}$ (see APPENDIX.1) .Let's determine the average throughput of $\mathrm{AP}_{1}$ which is calculated as follows:

$$
\begin{gathered}
=\frac{B_{D}}{N} \int_{r_{\min }}^{r_{\max }} \sum_{p=1}^{R_{D, \delta}(\rho)^{\operatorname{avg}}} \log _{2}(1 \\
\left.+\frac{N\left(\lambda_{p d} A_{p d}(m+1) h^{m_{k}+1} g\left(\phi_{k, \delta}\right)\right)^{2} P_{D}{ }^{2} e^{\frac{-4 \pi p_{\min } B_{D}}{N \omega_{c}}}}{4 \pi^{2}(N-2) \gamma^{2} N_{0}\left(r_{k, \delta}{ }^{2}+{h_{k}}^{2}\right)^{m+3}}\right)
\end{gathered}
$$$$
=\frac{B_{D}}{N \ln (2)} \int_{r_{\min }}^{r_{\max }} \sum_{p=1}^{p_{\min }} \ln \left(\rho U e^{\frac{-4 \pi p_{\min } B_{D}}{N \omega_{c}}}\right)-(m
$$$$
\text { +3) } \ln \left(r_{k, \delta}^{2}+h_{k}^{2}\right)
$$$$
=\frac{B_{D} r_{\max } p_{\min }}{N \ln (2)}\left[\ln \left(\rho_{0} U e^{\frac{-2 \pi p_{\min } B_{D}}{N \omega_{c}}}\right)-(m+\right.
$$$$
\text { 3) }\left(\left(\ln \left(r_{\max }^{2}+h_{k}^{2}\right)-2\right)+\right.
$$$$
\left.\left.\left(2 \frac{h_{k}}{r_{\max }} \tan ^{-1}\left(\frac{r_{\max }}{h_{k}}\right)\right)\right)\right](23)
$$

Where $\rho_{0}=\frac{P_{D}{ }^{2}}{B_{D}}$ is the optimization parameter which can be adapted with the system structure requirements for a minimum given number of subcarriers in each region, whereas its value is limited $0<\rho_{0}<1$ and $U=$ $\frac{N\left(\lambda_{p d} A_{p d}(m+1) h^{m_{k}+1} g\left(\emptyset_{k, \delta}\right)\right)^{2}}{4 \pi^{2}(N-2) \gamma^{2} N_{0}}$. After determining the average throughput in terms of the optimization parameter $\rho_{0}$ and the minimum of the subcarriers to be allocated to the UEs in different regions of $\mathrm{AP}_{1}$, we

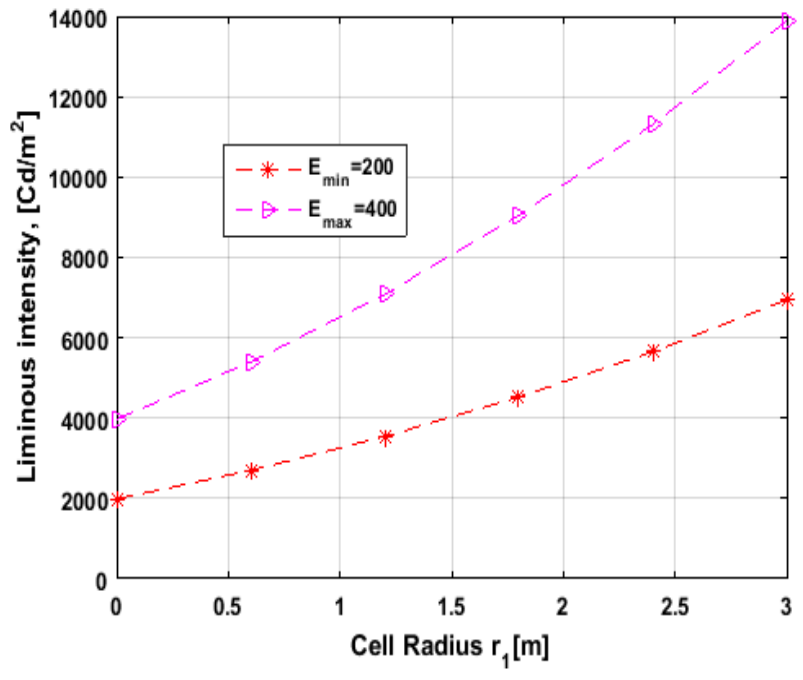

Figure. 14 The variation of the intensity in different regions of the coverage area $\mathrm{AP}_{1}$

derive the average throughput $\mathrm{R}^{\mathrm{avg}}{ }_{\mathrm{D}, \delta}$ with respect to $\rho_{0}$ which was obtained in Eq. (23) in order to find the optimal value of the $\rho_{0}$ that maximizes the throughput of the edge user. In addition, an algorithm should be used to optimize this parameter which leads to a variation in the subcarriers power. This means that unequal power will be shared among the users contrary to what was assumed in the beginning of the analysis. The optimum $\rho_{0}$ can be defined as follows:

$$
\frac{\partial R_{D, \delta}{ }^{a v g}}{\partial \rho_{0}}=\frac{B_{D} r_{\max } p_{\min }}{N \ln (2)} \times \frac{1}{\rho_{0}}>0
$$

It is worth noting that the optimal value of $\rho_{0}$ depends not only on the radius of the cell but also on the error probability at the instantaneous $\mathrm{SNR}_{\mathrm{D}, \delta}$.In this case, the maximization of the average throughput which was obtained by calculating the derivation of Eq. (23) with respect to $\rho_{0}$ that can be rewritten as follows:

$$
\begin{aligned}
& \max _{\rho_{0}} R_{D, \delta}=\lim _{\rho_{0} \rightarrow 1} R\left(\rho_{0}\right)_{D, \delta} \times \\
& \operatorname{Pr}\left(z \times \overline{S N R\left(\rho_{0}\right)_{D, \delta}}>\operatorname{SNR}\left(\rho_{0}\right)_{D, \delta}\right)
\end{aligned}
$$

Where

$$
P_{z}=\operatorname{Pr}\left(z \times \overline{\operatorname{SNR}\left(\rho_{0}\right)_{D, \delta}}>\right.
$$
$\left.\operatorname{SNR}\left(\rho_{0}\right)_{D, \delta}\right)$ is the error probability under the AWGN channel and it is conditioned by the channel variation $\mathrm{z}$

$z=\frac{\left|H_{k, \delta}\right|^{2}}{\sqrt{E\left(\left|H_{k, \delta}\right|^{2}\right.}}, \overline{\operatorname{SNR}\left(\rho_{0}\right)_{D, \delta}}$ is the average signal noise-ratio. In addition, the condition written in Eq. (25) should be kept in order that the error probability can drop rapidly once the instantaneous $\overline{\operatorname{SNR}\left(\rho_{0}\right)_{D, \delta}}$ surpasses $\operatorname{SNR}\left(\rho_{0}\right)$.The error probability over the AWGN is thus given: 


$$
\begin{aligned}
P_{z} & =\operatorname{Pr}\left(z \times \overline{\operatorname{SNR}\left(\rho_{0}\right)_{D, \delta}}>\operatorname{SNR}\left(\rho_{0}\right)_{D, \delta}\right) \\
& =\int_{Z}^{+\infty} p_{z}(x) d x
\end{aligned}
$$

Where $\quad p_{z}(x)=\frac{1}{\sigma \sqrt{2 \pi}} \exp \left(\frac{-[x]^{2}}{2 \sigma^{2}}\right) \quad$ is the probability density function (PDF) of the Gaussian distribution.

As stated above, the uplink analysis wasn't taken into account in this investigation concerning the feedback rate and the amount of information, whereas its vital role will be next employed in order to know the UE state in each region through sending the full description of the instantaneous channel to AP. Fig. 15 describes the process of the throughput enhancement especially in the edge of the AP, in which the LS regression [32] is employed to find the estimated requested throughput $\widehat{\mathrm{R}_{\text {req }}}$ relying on the optimal value of $\rho_{0}$ and the value of $p_{\min }$. Algorithm 1 describes the main steps to evaluate the behavior of the throughput with respect to the optimal value of $\rho_{0}$.

Algorithm 1-Throughput enhancement based on optimal solution

1. Input: $N, B_{d}, P_{D}, \rho$;

2. Initialization: $\mathrm{r}_{\mathrm{k}}=0$;

3. for $0 \leq \mathrm{p} \leq \mathrm{N} / 2-1$

Throughput calculation using Eq. (22)

4. update $r_{k}=r_{k}+0.1$

if $\mathrm{SNR}_{\mathrm{D}, \delta, \mathrm{P}} \leq \mathrm{SNR}_{\mathrm{T}}$

$$
\begin{aligned}
\max _{\rho_{0}} R_{D, \delta} & =\lim _{\rho_{0} \rightarrow 1}\left(\mathrm{R}\left(\rho_{0}\right)_{\mathrm{D}, \delta}\right) \operatorname{Pr}\left(z \times \overline{S N R_{D, \delta}}\left(\rho_{0}\right)>S N R_{D, \delta}\left(\rho_{0}\right)\right. \\
& \text { Else } \mathrm{R}^{a v g}{ }_{\mathrm{D}, \delta}=\mathrm{R}_{\mathrm{D}, \delta} \\
& \text { End if } \\
& \text { End for }
\end{aligned}
$$

\subsection{Simulations results and performance discussion of the proposed method}

The optical wireless network parameters that are employed in this study are enumerated below in Table. 1as default parameters. A large empty room is typically designed to be a work space with a size of $10 \times 10 \times 3 \mathrm{~m}$. According to the results shown in Fig. 5 ,a $\psi_{1 / 2}$ of $60^{\circ}$ is chosen to be the adequate value for lightning performance. The UE is elevated $0.85 \mathrm{~m}$ from the earth's surface while the vertical distance between the UE and the AP is determined at $2.15 \mathrm{~m}$. $A_{p d}$ of $1 \mathrm{~cm}^{2}$ is the reasonable value in the optical
Table 1. System settings

\begin{tabular}{|c|c|c|}
\hline Parameter & Symbol & Value \\
\hline $\begin{array}{c}\text { Vertical distance between the UE } \\
\text { and AP }\end{array}$ & $\mathrm{h}_{\mathrm{k}}$ & $2.15 \mathrm{~m}$ \\
\hline $\begin{array}{c}\text { Coverage area radius } \\
\text { Number of subcarriers }\end{array}$ & $r_{k}$ & $3 \mathrm{~m}$ \\
\hline Downlink Bandwidth & $\mathrm{B}_{\mathrm{d}}$ & $5 \mathrm{MHz}$ \\
\hline $\begin{array}{c}\text { Transmitted optical power } \\
\text { "Downlink" }\end{array}$ & $P_{D, o}$ & $4 \mathrm{~W}$ \\
\hline $\begin{array}{c}\text { Area of the detector } \\
\text { Fitted coefficients of the LED }\end{array}$ & $\mathrm{A}_{\mathrm{pd}}$ & $1 \mathrm{~cm}_{c}$ \\
\hline PD responsivity & $\lambda_{p d}$ & $15.5 \mathrm{Mrad}$ \\
\hline $\mathrm{s}$
\end{tabular}

attocell network. As shown in Fig. 7 a good tradeoff between the modulation bandwidth and the other bandwidth is provided by the chosen LED. Due to using the blue filter at the UE, the PD responsivity of $0.1 \mathrm{~A} / \mathrm{W}$ is chosen to be the suitable value to obtain a better response of the PD According to Eq. (19), Fig. 16 presents the results of the SINR likelihood based on the two APs, the first for the area coverage $\left(\mathrm{AP}_{1}\right)$ and the second for the interference $\left(\mathrm{AP}_{2}\right)$, whereas differences exist in the distribution of the SINR. Let's analyze the system performance with different values of $\Delta$. Firstly, with $\Delta=1$ the $-9.8 \mathrm{~dB}$ exhibits the worst value of the SINR owing to the fact that edge users employ the same channels which increase by $\sqrt{\Delta}$. Secondly, the SINR is shifted to the right by $5 \mathrm{~dB}$ when $\Delta=2$ is employed. Finally, the system shows enhancements in terms of SINR boost SINR $=6.8 \mathrm{~dB}$ through using $\Delta=3$.

It is worth clarifying the method followed in Fig. 15 before explaining the results of the proposed method. In doing so, the AP determines a threshold for all UEs in different regions while each UE compares its SNR to this threshold. The value of the threshold is chosen to be $\varepsilon=-15 \mathrm{~dB}$ as a minimum value of the SNR. In this case, when the SNR is inferior to the threshold, the UE sends information concerning the received optical power in terms of the SNR .Thus applying algorithm 1.In other words, Fig. 17 depicts the comparison between the enhanced throughput of the $\mathrm{AP}_{1}$ in the four different values of the $\rho_{0}$ by using Eq. (25). As we have shown above, the UEs were uniformly distributed in each region. However, we will only focus on certain radius $r_{1,1}=0 \mathrm{~m}, r_{2,1}=1 \mathrm{~m}, r_{3,1}=3 \mathrm{~m}$ where the UEs will be set up .Obviously, we notice an enhancement in terms of 


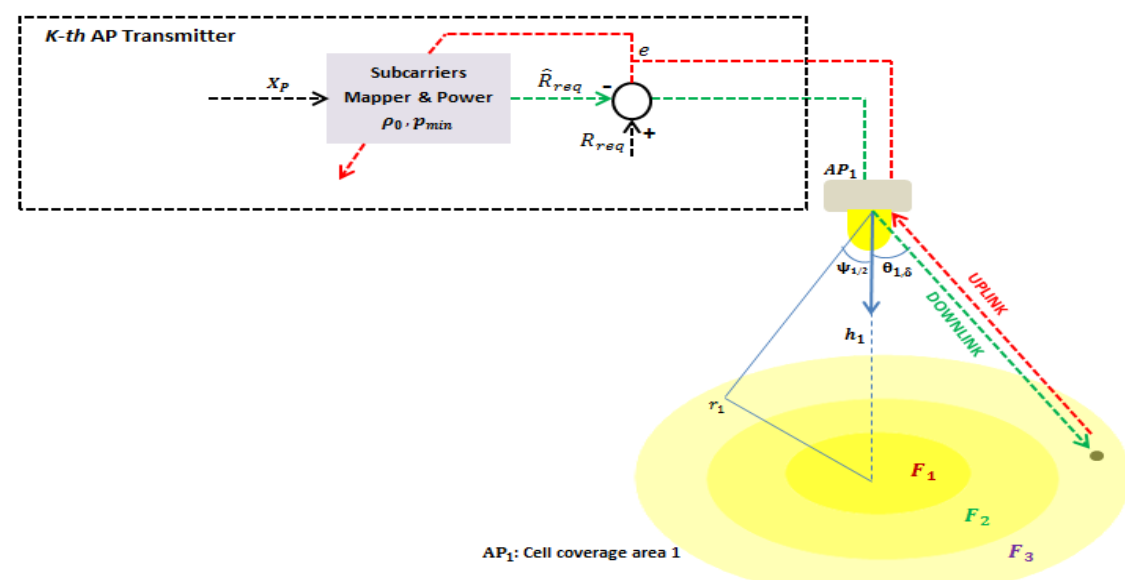

Figure. 15 Throughput enhancement based on the optimization method

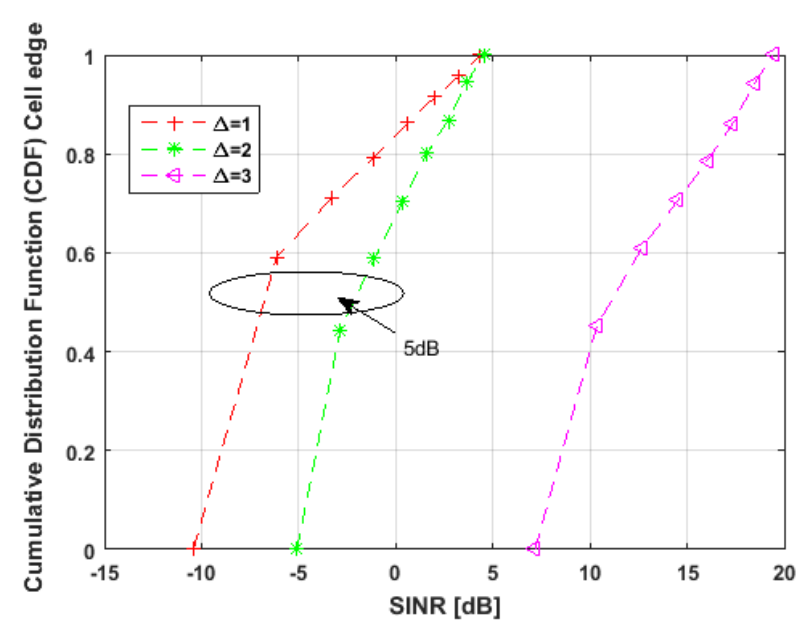

Figure. 16 The effective downlink SINR distribution between the different values of the FFR at the cell edge

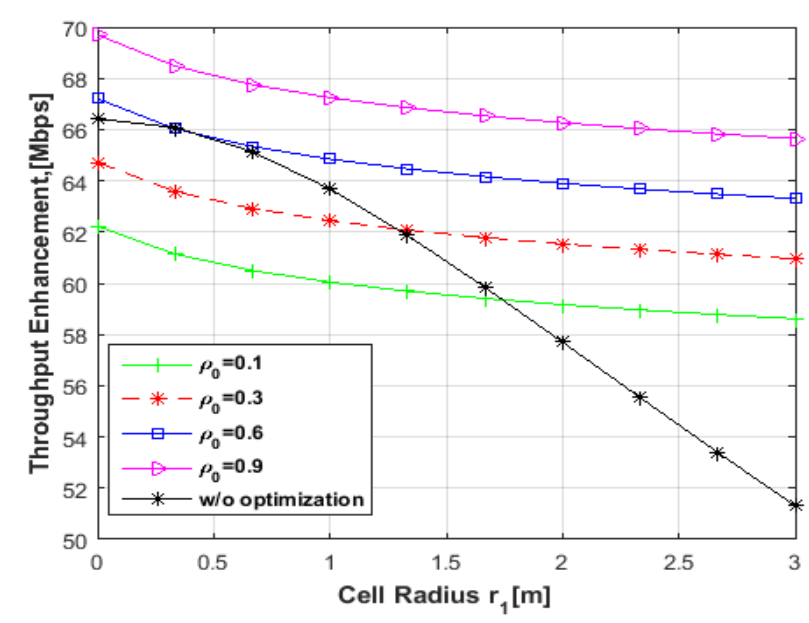

Figure. 17 The enhanced throughput based on the powerbandwidth trade-off

the throughput owing to the values change of the $\rho_{0}$.It can also be noticed from these results that the maximum throughput $\mathrm{R}\left(\rho_{0}\right)_{\mathrm{D}, \delta}=70 \mathrm{Mbips}$ at $r_{1,1}=0 \mathrm{~m}$ corresponds to an optimal value $\rho_{0}=0.9$

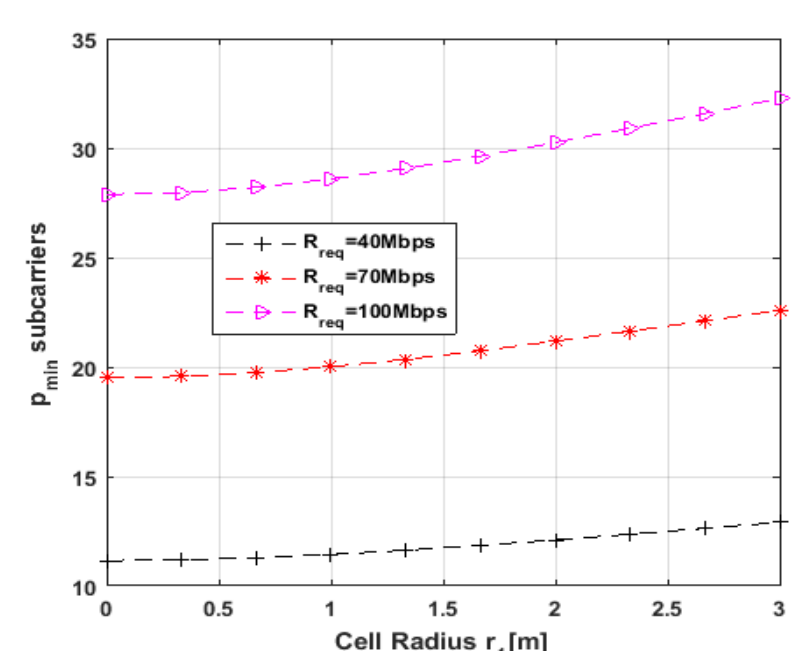

Figure. 18 Minimum number of subcarriers versus the $\mathrm{AP}_{1}$ radius

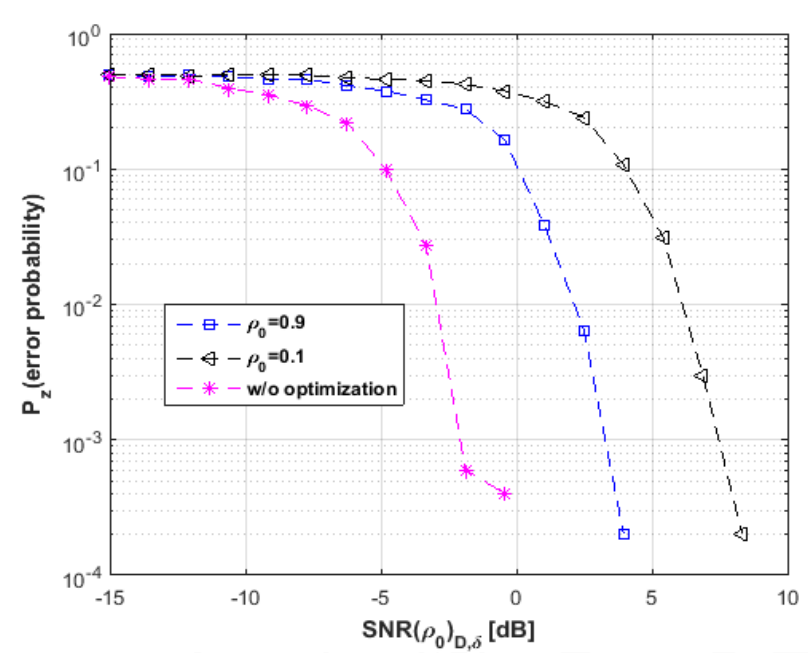

Figure. 19 Behavior of error probability versus SNR of a $\rho_{0}$ changement

along with the minimum throughput $R\left(\rho_{0}\right)_{D, \delta}=65 \mathrm{Mbps}$ at $\mathrm{r}_{3,1}=3 \mathrm{~m}$. However, the other values of the $\rho_{0}$ show better improvements at the 
throughput compared with the throughput without optimization (w/o optimization). Fig. 18 illustrates the minimum number of subcarriers needed to be allocated in each region while keeping the optimum value of $\rho_{0}=0.9$ fixed. We can clearly notice that when the requested throughout is increased $\left(\mathrm{R}\left(\rho_{0}\right)_{\mathrm{D}, \delta}=100 \mathrm{Mbps}\right)$, the minimum number of subcarriers is increased $\left(\mathrm{p}_{\min }=27\right)$. With regard to the targeted value which is $R\left(\rho_{0}\right)_{D, \delta}=70 \mathrm{Mbps}$, the minimum subcarriers at $r_{1,1}=0 \mathrm{~m}$ are $p_{\min }=19$, whereas at $r_{1,1}=3 \mathrm{~m} \mathrm{p} p_{\min }=22$. Finally, Fig. 19 represents the curves performance in terms of minimum and maximum value of $\rho_{0}$. We can also observe that $\operatorname{SNR}\left(\rho_{0}=0.1\right)_{D, \delta}=-4 \mathrm{~dB}$ is the minimum value where the curve starts falling to achieve a minimum error probability $\left(P_{z} \cong 10^{-4}\right)$, whereas the $\operatorname{SNR}\left(\rho_{0}=0.9\right)_{D, \delta}=8 \mathrm{db}$ shows a better result $\left(P_{z} \cong 10^{-4}\right)$ compared with the other curves. Nevertheless, the error probability (w/o optimization) noticeably shows better performance at the expense of $-3.54 \mathrm{~dB}$ reduction in $\operatorname{SNR}\left(\rho_{0}\right)_{D, \delta}$ as revealed in Fig. 19 .We further observe that the combination $\left(\rho_{0}, p_{\min }\right)$ seeks to improve the performance of the $\mathrm{UE}$ at the different values of the AP radius. Subsequently, the simulations results show that the lower $\rho_{0}$ underestimates both the throughput and system performance in terms of $S N R\left(\rho_{0}\right)_{D, \delta}$, whereas the higher value of $\rho_{0}$ improves the system performance. Subsequently, the simulations results show that the lower $\rho_{0}$ underestimates both the enhanced throughput and system performance in terms of SNR, whereas the higher value of $\rho_{0}$ improves the system performance.

\section{Conclusion}

In this study, we sought to strike a balance between power and bandwidth within the framework of enhancing the throughput of the edge UEs in the optical attocell network, whereas the throughput was maximized with $\rho_{0}=0.9\left(\mathrm{R}\left(\rho_{0}\right)_{\mathrm{D}, \delta}=70 \mathrm{Mbps}\right)$ at $\mathrm{r}_{1,1}=0 \mathrm{~m}$.In addition, the throughput of the edge UEs knew noticeable improvement, while the system performance in terms of the throughput reaches at $\mathrm{r}_{1,1}=0 \mathrm{~m}$ to $\mathrm{R}\left(\rho_{0}\right)_{\mathrm{D}, \delta}=65 \mathrm{Mbps}$ compared with the throughput without optimization $\mathrm{R}_{\mathrm{D}, \delta}=52 \mathrm{Mbps}$. The $\mathrm{AP}$, on the one hand, jointly optimized the power and bandwidth in order to achieve the QoS satisfaction in terms of the lower error probability and, on the other hand, maximized the system throughput as mentioned earlier. A deep theoretical analysis was firstly considered to be the policy towards extracting the closed expressions. Geometrical solution and FFR scheme were the key enabling elements to mitigate the ICI issue while three regions were defined in each AP along with priority regions. It was also shown that the optimization with different values of $\rho_{0} \quad\left(\rho_{0}=0.1,0.3,0.3\right)$ led to better downlink throughput $\left(\mathrm{R}\left(\rho_{0}\right)_{\mathrm{D}, \delta}=58 \mathrm{Mbps}, 60 \mathrm{Mbps}, 63 \mathrm{Mbps}\right)$ compared with the throughput without optimization. Our future studies will be directed to analyzing the overall throughput system (downlink- uplink) even if the uplink wasn't robustly taken into account in this study but it served the AP to know the state channel.

\section{Appendix. 1}

Regarding Eq. (22) and Fig. 11, three UEs are located at different values of the radius $\left(r_{1,1}, r_{2,1}, r_{3,1}\right)$, whereas the radius at the illumination center is $r_{1,1}=0$. The AP dynamically allocates $\rho_{\text {min }}$ subcarriers to the UE to match its QoS in terms of the downlink throughput, thus $\mathrm{R}_{\mathrm{D}, \delta}\left(\mathrm{r}_{\mathrm{k}}\right)=\mathrm{R}_{\text {req }} \cdot$ We have:

$$
\begin{aligned}
& R_{\text {req }}=\frac{B_{d}}{N} \sum_{p=1}^{p_{\min }} \log _{2}\left(\frac{\rho U e^{\frac{-4 \pi p_{\min } B_{D}}{N w_{c}}}}{\left(r_{k, \delta}{ }^{2}+{h_{k}}^{2}\right)^{m+3}}\right) \\
& =\frac{B_{d}}{N} \sum_{p=1}^{p_{\min }} \log _{2}\left(\frac{\rho U}{\left(r_{k, \delta}{ }^{2}+{h_{k}}^{2}\right)^{m+3}}\right) \\
& +\frac{B_{d}}{N} \sum_{p=1}^{p_{\min }} \log _{2}\left(e^{\frac{-4 \pi p_{\min } B_{D}}{N w_{C}}}\right) \\
& =\frac{p_{\min } B_{D}}{N} \log _{2}\left(\frac{\rho U}{\left(r_{k, \delta}{ }^{2}+{h_{k}}^{2}\right)^{m+3}}\right) \\
& -\frac{4 \pi}{w_{C}}\left(\frac{B_{D}}{N}\right)^{2} \log _{2}(e) \sum_{p=1}^{p_{\min }} p_{\text {min }} \\
& =\frac{p_{\min } B_{D}}{N} \log _{2}\left(\frac{\rho U}{\left(r_{k, \delta}{ }^{2}+{h_{k}}^{2}\right)^{m+3}}\right) \\
& -\frac{2 \pi}{w_{C}}\left(\frac{B_{D}}{N}\right)^{2} \log _{2}(e) p_{\text {min }}\left(p_{\text {min }}\right. \\
& +1 \text { ) } \\
& \Rightarrow p_{\text {min }}{ }^{2}+\left(1-\frac{\log _{2}\left(\frac{\rho U}{\left(r_{k, \delta}^{2}+h_{k}^{2}\right)^{m+3}}\right)}{\frac{2 \pi}{w_{C}}\left(\frac{B_{D}}{N}\right) \log _{2}(e)}\right) p_{\text {min }} \\
& +\frac{R_{r e q}}{\frac{2 \pi}{w_{C}}\left(\frac{B_{D}}{N}\right)^{2} \log _{2}(e)}=0
\end{aligned}
$$

After obtaining the above quadratic equation, the solutions of $p_{\min }$ can be found as follows: 


$$
\begin{gathered}
p_{\min }=\frac{\left(\frac{X}{Y}-1\right)-\sqrt{\left(\frac{X}{Y}-1\right)^{2}-\frac{4 R_{\text {req }}}{Z}}}{2} \\
=\frac{1}{2}\left(\frac{X}{Y}-1\right)\left(1-\left(1-\frac{\frac{4 R_{\text {req }}}{Z}}{\left(\frac{X}{Y}-1\right)^{2}}\right)^{1 / 2}\right)
\end{gathered}
$$

Where $X=\log _{2}\left(\frac{\rho U}{\left(r_{\left.k, \delta^{2}+h_{k}{ }^{2}\right)^{m+3}}\right.}\right)$,

$$
\begin{aligned}
& Y=\frac{2 \pi}{w_{c}}\left(\frac{B_{D}}{N}\right) \log _{2}(e) \\
& Z=\frac{2 \pi}{w_{c}}\left(\frac{B_{D}}{N}\right)^{2} \log _{2}(e) \text { and } \frac{X}{Y} \gg 1
\end{aligned}
$$

The binomial approximation $(1+x)^{n} \cong 1+n x$ for small $x$ is employed in order to obtain the approximate number of subcarriers .Hence, this latter can be rewritten as follows:

$$
p_{\text {min }}=\frac{\ln (2) N R_{r e q}}{B_{D} \ln (\rho U)-m+3 \ln \left(r_{k, \delta}{ }^{2}+{h_{k}}^{2}\right)}
$$

\section{Notation list}

K-th: number of the APs.

$\delta$-th: number of the UE or receiver.

p-th: number of the subcarriers.

$\rho_{0}$ : optimized parameter.

$P_{Z}$ : the error probability.

$\mathrm{R}_{\mathrm{D}, \delta}$ : throughput of UE in downlink sid.

$R_{D, \delta}{ }^{a v g}$ : the average throughput of the UE in downlink side.

$R\left(\rho_{0}\right)_{D, \delta}=$ the enhanced throughput.

$\mathrm{SNR}_{\mathrm{D}, \delta}$ : is the average signal-noise-ratio of the UE in the downlink side.

$\overline{\mathrm{SNR}_{\mathrm{D}, \delta}}$ : is the average signal -noise-ratio.

$\operatorname{SNR}\left(\rho_{0}\right)_{D, \delta}$ : is the average signal -noise-ratio with the optimized parameter of the UE in the downlink side.

\section{Conflicts of Interest}

The authors whose names are listed immediately above certify that they have no affiliations with or involvement in any organization or conflict of interest.

\section{Author Contributions (Mandatory)}

The entire work of conceptualization, formal analysis, validation, and writing, editing and modification of article were done by Abdelmoujoud Assabir under the control of Jamal Elmhamdi.

\section{Acknowledgments}

Under the current circumstances of the COVID19 , we gratefully acknowledge support for this work from National Center for Scientific and Technical Research of Rabat-Morocco.

\section{References}

[1] S. JOSE and Calif, "Cisco Mobile Visual Networking Index (VNI) Forecast Projects 7Fold Increase in Global Mobile Data Traffic from 2016-2021", https://newsroom.cisco.com/press-releasecontent ?articleId $=1819296,2017$.

[2] M. Shafi, A. F. Molisch, P. J. Smith, T. Haustein, P. Zhu, P. D. Silva, F. Tufvesson, A. Benjebbour, and G. Wunder, "5g: A tutorial overview of standards, trials, challenges, deployment, and practice", IEEE Journal on Selected Areas in Communications, Vol. 35, No. 6, pp. 1201-1221, 2017.

[3] H. Alshaer and H. Haas, "Bidirectional LiFi Attocell Access Point Slicing Scheme", IEEE Transactions on Network and Service Management, Vol. 15, No.3, pp. 909-922, 2018.

[4] X. Guo and N. Chi, "Superposed 32QAM Constellation Design for $2 \times 2$ Spatial Multiplexing MIMO VLC Systems", Journal of Lightwave Technology, Vol. 38, No. 7, pp. 17021711, 2020.

[5] M. Z. Chowdhury, M. T. Hossan, A. Islam, and Y. M. Jang, "A comparative survey of optical wireless technologies: Architectures and applications", IEEE Access, Vol. 6, pp. 98199840, 2018.

[6] S. Al-Ahmadi, O. Maraqa, M. Uysal, and S. M. Sait, "Multi-User Visible Light Communications: State-of-the-Art and Future Directions", IEEE Access, Vol. 6, pp. 7055570571, 2018.

[7] S. K. Wilson and J. Holliday, "Scheduling methods for multi-user optical wireless asymmetrically-clipped", Journal of Communication and Networks, Vol. 13, No. 13, pp. 655-663, Dec.2011.

[8] D. S. Mohammad, X. Wu, M. Safari, and H. Haas, "Bidirectional User Throughput Maximization Based on Feedback Reduction in LiFi Networks", IEEE Transaction on Communication, Vol. 66, No. 7, pp. 3172-3186, 2018.

[9] P. Pattanayak and P. Kumar, "SINR based Limited Feedback Scheduling for MIMOOFDM heterogeneous broadcast networks", In: 
Proc.of National Conf. on Communication (NCC), Guwahati, India, 2016.

[10] D. Bykhovsk and S. Arnon, "Multiple Access Resource Allocation in Visble Light Communication Systems", Journal of Lightwave Technology, Vol. 32, No. 8, pp. 1594 - 1600, 2014.

[11] R. K. Mondal, Nirzhar Saha, N. T. Le and Y. M. Jang, "SINR-Constrained Joint Sheduling and Optimal Ressource Allocation in VLC Based WPAN System", Wireless Personal Communication, Vol. 78, No. 4, pp. 1935-1951, 2014.

[12] F. Jin X. Li, R. Zhang, C. Dong and L. Hanzo, "Resource Allocation Under delay - Guarantee Constraints for Visible-light Communication", IEEE Access, Vol. 4, pp. 7301-7312, 2016.

[13] M. Z. Chowdhury, M. Shahin Uddin, and Y. Mi. Jang, "Dynamic Channel Allocation for ClassBased QoS Provisioning and Call Admission in Visible Light Communication", Arabian Journal for Science and Engineering, Vol. 39, pp. 1007-1016, 2013.

[14] B. Ghimire and H. Haas, "Self-organising interference coordination in optical wireless networks", EURASIP Journal on Wireless Communications, No. 131, 2012.

[15] L. Chen, W. Wang, and C. Zhang, "Coalition Formation for Interference Management in Visisble Light Communication Networks", IEEE Transactions on Vehicular Technology, Vol. 66, No. 8, pp. 7278- 7285, 2017.

[16] H. Kazemi and H. Haas, "Downlink cooperation with fractional frequency reuse in DCOOFDMA optical attocell networks", In: Proc. of International Conf. on Communications (ICC), Kuala Lumpur, Malaysia, 2016.

[17] H. Kazemi, M. Safari, and H. Haas, "Spectral Efficient Coorperative Downlink Transmission Schemes for DCO-OFDM-Based Optical Attocell Networks", In: Proc. of Vehicular Technology Conference (VTC-Fall), Montreal, QC, Canada, 2016.

[18] L. Chen, W. Wang, and C. Zhang, "Coalition Formation for Interference Management in Visisble Light Communication Networks", IEEE Transactions on Vehicular Technology, Vol.66, No.8, pp. 7278 - 7285, 2017.

[19] A. A. Dowhuszko, A. I. Pérez-Neira, "Achievable data rate of coordinated multi-point transmission for visible light communication", In: Proc.of Annual International Symposium on Personal, Indoor, and Mobile Radio Communications (PIMRC), Montreal, QC, Canada, 2017.
[20] J. Fakidis, D. Tsonev and H. Haas, "A comparison between DCO-OFDMA and synchronous one-dimensional OCDMA for optical wireless communications", In: Proc.of Annual International Symposium on Personal, Indoor, and Mobile Radio Communications (PIMRC), London, UK, 2013.

[21] T. Komine and M. Nakagawa, "Fundamental analysis for visible-light communication system using LED lights", IEEE Transactions on Consumer Electronics, Vol. 50, No. 1 pp. 100 107. June 2004.

[22] M. F. Keskin, E. Gonendik, and S. Gezici, "Improved lower bounds for ranging in synchronous visible light positioning systems", Journal of Lightwave Technology, Vol. 34, No. 23, pp. 5496-5504, 2016.

[23] H. Qian, S. J. Yao, S. Z. Cai, and T. Zhou, "Adaptive Postdistortion for Nonlinear LEDs in Visible Light Communication", IEEE Photonics Journal, Vol. 6, No. 4, 2014.

[24] A. Abdelmoujoud, J. Elmhamdi, and A.H ammouch, "Mitigation of the Memory-less Nonlinearity Based on Adaptive Technique", International Review on Modelling and Simulations, Vol. 12, No. 2, 2019.

[25] H. Le Minhet al, “100-Mb/s NRZ Visible Light Communications Usinga Postequalized White LED”, IEEE Photon, Technol, Letter, Vol. 21, No. 15, pp. 1063-1065, 2009.

[26] M. Bacha, M. D. Renzo, and B. Clerckx, "Treating Interference as Noise in Cellular Networks: A Stochastic Geometry Approach", IEEE Transactions on Wireless Communications, Vol. 19, No. 3, pp. 1918-1932, 2020.

[27] K. Koufos and C. P. Dettmann, "Temporal Correlation of Interference and Outage in Mobile Networks over One-Dimensional Finite Regions", IEEE Transactions on Mobile Computing, Vol. 17, No. 2, pp. 475 - 487, 2018.

[28] Y. Ben Ezra and B. I. Lembrikov, "Application of All-Optical Memory for Advanced Modulation Formats in Communication IntraDatacenter Networks (Intra-DCNs)", In: Proc. of International Conf. on Transparent Optical Networks, Bucharest, Romania, 2018.

[29] T. S ugitani, T. Kitazawa, and W. Uemura, "About Lighting Level of the Visible Light Communication", In: Proc. of Global Conf. on Consumer Electronics (GCCE), Nara, Japan, 2018.

[30] P. A. Haigh, P. Chvojka, S. Zvánovec, Z. Ghassemlooy, and I. Darwazeh, "Analysis of Nyquist Pulse Shapes for Carrierless Amplitude 
and Phase Modulation in Visible Light Communications", Journal of Lightwave Technology, Vol. 36. No. 20, pp. 5023- 5029, 2018.

[31] A. Adnan, Y. Liu, C. W. Chow, and C. G. Yeh "Demonstration of Non-Hermitian Symmetry (NHS) IFFT/FFT Size Efficient OFDM NonOrthogonal Multiple Access (NOMA) for Visible Light Communication", IEEE Photonics Journal, Vol. 12, No. 3, 2020.

[32] A. Saenmuang and S. Sitjongsataporn, "Convergence and Stability Analysis of Spline Adaptive Filtering based on Adaptive Averaging Step-size Normalized Least Mean Square Algorithm", International Journal of Intelligent Engineering systems, Vol. 13, No. 2, pp. 267277, 2020. 